\title{
Restoring the Molecular Clockwork within the Suprachiasmatic Hypothalamus of an Otherwise Clockless Mouse Enables Circadian Phasing and Stabilization of Sleep-Wake Cycles and Reverses Memory Deficits
}

\author{
${ }^{\circledR}$ Elizabeth S. Maywood, ${ }^{1}{ }^{\circledR}$ Johanna E. Chesham, ${ }^{1}$ Raphaelle Winsky-Sommerer, ${ }^{2}$ and ${ }^{\circledR}$ Michael H. Hastings ${ }^{1}$ \\ ${ }^{1}$ Division of Neurobiology, Medical Research Council Laboratory of Molecular Biology, Cambridge CB2 0QH, United Kingdom, and ${ }^{2}$ Surrey Sleep \\ Research Centre, Faculty of Health and Medical Sciences, University of Surrey, Guildford GU2 7XP, United Kingdom
}

The timing and quality of sleep-wake cycles are regulated by interacting circadian and homeostatic mechanisms. Although the suprachiasmatic nucleus $(\mathrm{SCN})$ is the principal clock, circadian clocks are active across the brain and the respective sleep-regulatory roles of SCN and local clocks are unclear. To determine the specific contribution(s) of the SCN, we used virally mediated genetic complementation, expressing Cryptochromel (Cryl) to establish circadian molecular competence in the suprachiasmatic hypothalamus of globally clockless, arrhythmic male Cry1/Cry2-null mice. Under free-running conditions, the rest/activity behavior of Cry1/Cry2-null controls expressing EGFP $\left(\mathrm{SCN}^{\mathrm{Con}}\right)$ was arrhythmic, whereas Cryl-complemented mice $\left(\mathrm{SCN}^{\mathrm{Cryl}}\right)$ had coherent circadian behavior, comparable to that of Cryl,2-competent wild types (WTs). In $\mathrm{SCN}^{\mathrm{Con}}$ mice, sleep-wakefulness, assessed by electroencephalography (EEG)/electromyography (EMG), lacked circadian organization. In $\mathrm{SCN}^{\mathrm{Cryl}}$ mice, however, it matched WTs, with consolidated vigilance states [wake, rapid eye movement sleep (REMS) and non-REMS (NREMS)] and rhythms in NREMS $\delta$ power and expression of REMS within total sleep (TS). Wakefulness in $\mathrm{SCN}^{\mathrm{Con}}$ mice was more fragmented than in WTs, with more wake-NREMS-wake transitions. This disruption was reversed in $\mathrm{SCN}^{\mathrm{Cryl}}$ mice. Following sleep deprivation (SD), all mice showed a homeostatic increase in NREMS $\delta$ power, although the $\mathrm{SCN}^{\mathrm{Con}}$ mice had reduced NREMS during the inactive (light) phase of recovery. In contrast, the dynamics of homeostatic responses in the $\mathrm{SCN}^{\mathrm{Cryl}}$ mice were comparable to WTs. Finally, $\mathrm{SCN}^{\mathrm{Con}}$ mice exhibited poor sleep-dependent memory but this was corrected in $\mathrm{SCN}^{\text {Cryl }}$ mice. In clockless mice, circadian molecular competence focused solely on the SCN rescued the architecture and consolidation of sleep-wake and sleep-dependent memory, highlighting its dominant role in timing sleep.

Key words: circadian; clock; cryptochrome; hippocampus; NREM sleep; sleep homeostasis

Significance Statement

The circadian timing system regulates sleep-wake cycles. The hypothalamic suprachiasmatic nucleus (SCN) is the principal circadian clock, but the presence of multiple local brain and peripheral clocks mean the respective roles of SCN and other clocks in regulating sleep are unclear. We therefore used virally mediated genetic complementation to restore molecular circadian functions in the suprachiasmatic hypothalamus, focusing on the SCN, in otherwise genetically clockless, arrhythmic mice. This initiated circadian activity-rest cycles, and circadian sleep-wake cycles, circadian patterning to the intensity of non-rapid eye movement sleep (NREMS) and circadian control of REMS as a proportion of total sleep (TS). Consolidation of sleep-wake established normal dynamics of sleep homeostasis and enhanced sleep-dependent memory. Thus, the suprachiasmatic hypothalamus, alone, can direct circadian regulation of sleep-wake.

Received Dec. 10, 2020; revised June 29, 2021; accepted July 15, 2021

Author contributions: M.H.H. and E.S.M. designed research; E.S.M. and J.E.C. performed research; R.W.-S. contributed unpublished reagents/analytic tools; M.H.H., E.S.M., and J.E.C. analyzed data; E.S.M. wrote the first draft of the paper; M.H.H., E.S.M., and R.W.-S. edited the paper; M.H.H. and E.S.M. wrote the paper.

This work was supported the Medical Research Council Core Funding Grant MC_U105170643 (to M.H.H.). We thank the invaluable support of the MRC Laboratory of Molecular Biology Electronics and Mechanical Workshops, particularly Chris Palmer, Tom Pratt in the IT Department, and the Ares (Biological Services Group). We also thank Dr. X. Yu and Prof. W. Wisden, Imperial College, London, for the use of software for analyzing the NOR.
The authors declare no competing financial interests.

Correspondence should be addressed to Elizabeth S. Maywood at emaywood@mrc-Imb.cam.ac.uk or Michael H. Hastings at mha@mrc-Imb.cam.ac.uk.

https://doi.org/10.1523/JNEUROSCl.3141-20.2021

Copyright $\odot 2021$ Maywood et al.

This is an open-access article distributed under the terms of the Creative Commons Attribution 4.0 International license, which permits unrestricted use, distribution and reproduction in any medium provided that the original work is properly attributed. 


\section{Introduction}

The timing and quality of sleep are determined by a circadian process that ensures sleep occurs appropriately within the lightdark (LD) cycle and a homeostatic process that tracks sleep need during wakefulness (Borbély and Achermann, 1999; Borbély et al., 2016). Whereas the identity of the homeostat remains unknown, the hypothalamic suprachiasmatic nucleus (SCN), is conventionally thought to mediate circadian control (Saper et al., 2005). At the molecular level, the SCN clock consists of transcriptional/posttranslational feedback loops (TTFL) in which Period (Per) and Cryptochrome (Cry) genes are trans-activated by CLOCK and BMAL1 heterodimers (Takahashi, 2017). Following their accumulation over circadian day, the encoded Per and Cry proteins inhibit trans-activation, closing the loop, while their subsequent degradation over circadian night allows the cycle to recommence. This TTFL is entrained to solar time by rods, cones and melanopsin-containing retinal ganglion cells innervating the SCN (Berson et al., 2002; Hattar et al., 2002, 2003). In constant darkness (DD), the TTFL runs to its intrinsic approximately $24 \mathrm{~h}$ period. Importantly, the TTFL is active in all tissues, including brain regions that regulate the sleep/wake cycle and memory (Hastings et al., 2018). The question arises, therefore, as to whether circadian control of sleep is mediated uniquely by the $\mathrm{SCN}$, or do local brain and peripheral clocks also contribute? Beyond that, the influence of the circadian system (SCN and/or local clocks) on sleep-wake cycles remains to be established: does it only affect timing, or does it modify its temporal architecture and/or homeostatic responses (Gillette, 2004)?

Loss-of-function ablation has demonstrated the necessity of the SCN for circadian timing of sleep under DD, and its redundancy in global sleep homeostasis (Tobler et al., 1983; Mistlberger, 2005), although increased non rapid-eye-movement sleep (NREMS) in SCN-ablated mice suggests a broader role in sleep regulation (Easton et al., 2004). Loss-of-genetic function approaches have also been used to interrogate circadian sleep control. Per1/Per2-null mice have a defective TTFL and arrhythmic sleep-wake patterns under DD, but not LD (Shiromani et al., 2004). Moreover, sleep deprivation (SD) induces time of day-dependent expression of Per1 and Per2 in the forebrain (Franken et al., 2007; Curie et al., 2013), but Per1/Per2-null mice show normal homeostatic regulation of the daily amounts of waking, NREMS, or REMS (Shiromani et al., 2004). Conversely, CLOCK mutant mice show altered homeostatic regulation in the amount of sleep, with less NREMS in both LD and DD (Naylor et al., 2000). Finally, Cry1/ Cry2-null mice lack TTFL function and so have no circadian pattern to sleep-wake in DD conditions, but show higher levels of NREMS and electroencephalography (EEG) $\delta$ power (Wisor et al., 2002). Further, when sleep-deprived under a LD schedule, Cry1/ Cry2-null mice show less NREMS recovery than do wild-type (WT) mice, suggesting a requirement for Cry proteins in sleep homeostasis. Altogether, this highlights the disparity in the literature on the precise role(s) of the circadian system in regulating sleep/ wake cycles.

Untangling the anatomic (SCN, extra-SCN) and genetic (Per, Cry, Clock) contributions to sleep regulation has therefore been challenging for loss-of-function approaches. Interpretation of results from clock gene mutants is constrained because global mutations compromise both the SCN and local clocks. Furthermore, as transcription factors, their encoded proteins may have non-circadian roles. Equally, SCN ablations disrupt neural circuitry and may compromise non-circadian processes (Mistlberger, 2005). Therefore, we chose a gain-of-function approach. Behavioral arrhythmia in clock mutant mice can be rescued by SCN grafting (Sujino et al., 2003) or by virally mediated genetic complementation targeted toward the SCN (Fuller et al., 2008; Maywood et al., 2018). We therefore tested the hypothesis that a molecularly competent SCN clock would be sufficient to co-ordinate phasing and stabilization of sleep-wake cycles, leading to improved sleep-dependent memory in an otherwise clockless and arrhythmic mouse. To do this we used adeno-associated viral (AAV) vectors to express control EGFP or a Cry1::EGFP fusion targeted at the SCN of Cry1/Cry2-null mice, leaving local clocks across the brain and periphery dysfunctional.

\section{Materials and Methods}

\section{Animals and housing}

All experiments were conducted in accordance with the UK Animals (Scientific Procedures) Act of 1986, with local ethical approval (MRC LMB, AWERB). We used four- to six-month-old male WT mice and Cry1,Cry2 double knock-out (CDKO) mice (van der Horst et al., 1999), all on a C57/BL/6J genetic background. There were no significant differences in body weights at the time of surgery (WT $=30.5 \pm 1.6 \mathrm{~g}$; CDKO $=28.5 \pm 1.1 \mathrm{~g}, n=5,18$, respectively). Mice were housed individually and their activity patterns were monitored continuously using running-wheels. Food and water were provided ad libitum. Mice were entrained to a $12 / 12 \mathrm{~h}$ light/dim red light cycle (LD) for at least $10 \mathrm{~d}$ before transfer to a schedule of continuous dim red light (DD) for $14 \mathrm{~d}$ for assessment of (ar)rhythmicity (DD1). Following surgery (see below), mice were maintained on a 12L:12D photoschedule for recovery before transfer to a second period of DD (DD2). In all of our studies, Zeitgeber time (ZT)0 denotes the time of lights-on and ZT12 lights-off under LD, whereas circadian time (CT)0 denotes the start of subjective day, and CT12 denotes the start of subjective night in $\mathrm{DD}$, as evidenced by activity onset.

Stereotaxic injection of AAV vector and implantation of EEG/electromyography (EMG) transmitters

Mice were anaesthetized using isoflurane (induction 2-4\%; maintenance $1 \%$ ) with body temperature thermostatically controlled using a heating pad. Rimadyl was used for postoperative analgesia. Under aseptic conditions, the animals received bilateral stereotaxic injections $(0.3 \mu \mathrm{l} / \mathrm{site})$ into the SCN ( $\pm 0.25 \mathrm{~mm}$ medio-lateral to bregma, $5.5 \mathrm{~mm}$ deep to dural surface) of a pan-cellular AAV-1 vector encoding pCry1-Cry1::EGFP $\left(3.26 \times 10^{12} \mathrm{cg} / \mathrm{ml}\right)$ for circadian expression of Cry1::EGFP fusion, driven by its minimal promoter ( $\mathrm{SCN}^{\mathrm{Cryl}}, n=9$ ), or pCryl-EGFP control $\left(4.8 \times 10^{12} \mathrm{cg} / \mathrm{ml}\right.$; SCN ${ }^{\text {Con }}, n=9$; Maywood et al., 2013; Edwards et al., 2016). At the same time, a telemetric transmitter (TL11M2-F20-EET, Data Sciences International) connected to electrodes for continuous EEG and EMG recordings was implanted subcutaneously. Two screws were implanted above the dura $(+1.5 \mathrm{~mm}$ anterior to bregma and +1.7 $\mathrm{mm}$ lateral to bregma, the second $+1.0 \mathrm{~mm}$ anterior and $+1.7 \mathrm{~mm}$ lateral to lambda, i.e., over the right hemisphere) around which the electrodes for measuring the EEG were placed and secured using dental cement (RelyX Unicem 2 automix; Henry Schein Medical Animal Health). The two EMG leads were inserted into the trapezius muscle $\sim 5 \mathrm{~mm}$ apart and sutured in place (Hasan et al., 2011; Lang et al., 2011). All mice were allowed $10-14 \mathrm{~d}$ of recovery following surgery. To confirm AAV targeting of the SCN, at the conclusion of the study, mice were culled and the brains dissected, fixed in $4 \%$ paraformaldehyde in phosphate buffer, cryopreserved overnight in $20 \%$ sucrose in PBS and then sectioned $(40 \mu \mathrm{m})$ on a freezing sledge microtome (Bright Instruments). Confocal microscopy (Zeiss 780 inverted confocal system) of the native EGFP signal in control $\mathrm{SCN}^{\mathrm{Con}}$ and $\mathrm{SCN}^{\mathrm{Cry} 1}$ groups was used to identify successful targeting of the SCN. Brain sections were mounted onto slides and coverslipped using Vectashield Hardset mounting medium with DAPI (Vector Labs, RRID:AB_2336788). Cell counts in SCN sections were assessed by the ratio of EGFP-positive cells to DAPI-positive cells using ImageJ. Because of inefficient targeting, two of the nine animals were removed from further analysis in the $\mathrm{SCN}^{\mathrm{Cryl}}$ group and one from the $\mathrm{SCN}^{\mathrm{Con}}$ group. 
A

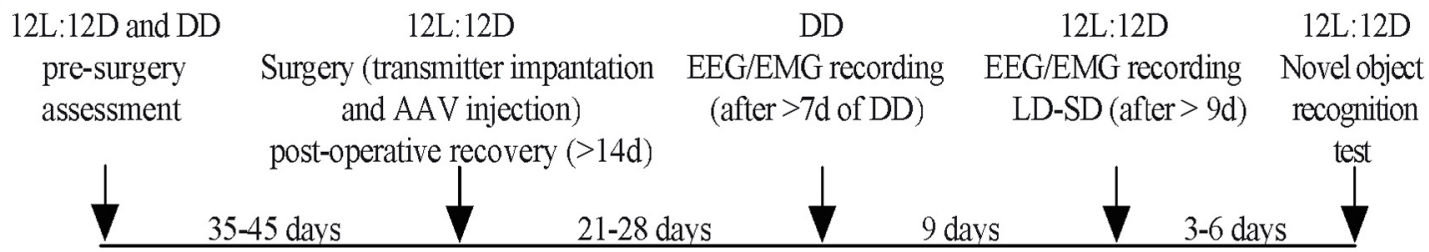

B

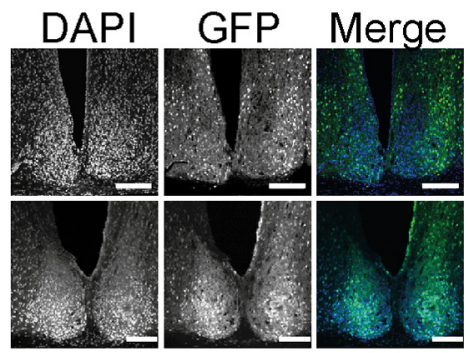

C



D

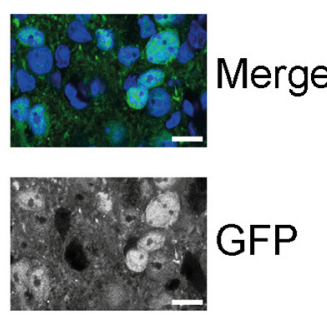

E

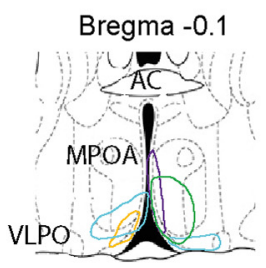

Bregma -0.46

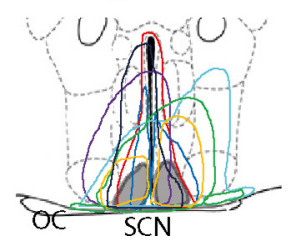

Bregma -0.94

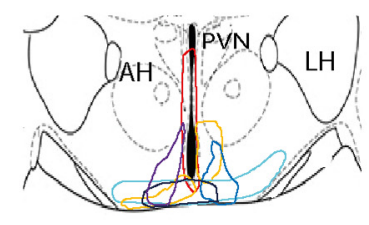

Overlay

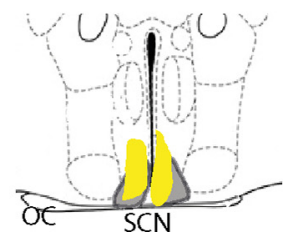

Figure 1. Histologic confirmation of the expression of pCry1-Cry1-EGFP in the suprachiasmatic hypothalamus of Cry1,2-null mice used in the sleep studies. $\boldsymbol{A}$, Timeline showing the order of experimental procedures and interventions. The number of days between each stage is listed. $\boldsymbol{B}$, Fluorescence confocal images from the brains of two mice injected with an AAV (pCry1-CRY1:: EGFP) to restore $\left(R Y 1\right.$ into the $S C N\left(S C N^{C r y 1}\right) ; 20 \times$; scale bar: $150 \mu \mathrm{m}$. C, Tiled $(4 \times 4)$ fluorescence confocal image from the same animal as in the upper panel of $\boldsymbol{B} ; 20 \times ;$ scale bar: $150 \mu \mathrm{m}$. $\boldsymbol{D}$, High-power fluorescence confocal image from the same animal as in the upper panel of $\boldsymbol{B}$, showing the nuclear localization of the GFP signal; $63 \times$; scale bar: $10 \mu \mathrm{m}$. $\boldsymbol{E}$, Color-coded depiction of the area of AAV-pCry1-CRY1::EGFP expression within the SCN and surrounding hypothalamus represented on coronal schematics modified from a mouse brain atlas (Paxinos and Franklin, 2001). Each color represents a single mouse used in the sleep studies. The gray shaded area represents the SCN. The yellow shaded area in the overlay plot shows the targeted area common to all mice. AC, anterior commissure; AH, anterior hypothalamus; MPOA, medial preoptic area; OC, optic chiasm; OT, optic tract; PVN, paraventricular nucleus; VLP0, ventrolateral preoptic nucleus.

\section{EEG/EMG recordings and determination of vigilance states and spectral analysis}

Transmitters were activated on the day before data collection and EEG/ EMG were recorded continuously from the freely moving animals in both DD ( $3 \mathrm{~d}$; $>21 \mathrm{~d}$ postsurgery; Fig. $1 A)$ and $\mathrm{LD}(2 \mathrm{~d}$; $>30-37 \mathrm{~d}$ postsurgery) using Data Sciences International hardware and Dataquest ART v2.3 Gold software. Vigilance states for consecutive 4-s epochs were classified by visual inspection according to standard criteria: wakefulness (high and variable EMG signal, low-amplitude EEG signal), NREMS (high EEG amplitude dominated by slow waves, low EMG), and REMS (low EEG amplitude, theta oscillations and muscle atonia). Vigilance states were analyzed offline using Neuroscore Software (v.2.1 Data Sciences International) with the EEG and EMG signals modulated with a high-pass $(3 \mathrm{~dB}, 0.5 \mathrm{~Hz})$ and a low-pass $(50 \mathrm{~Hz})$ analog filter and manually assessed. For both LD and DD conditions, continuous recordings were analyzed and time spent in each vigilance state was expressed as a percentage of the total recording time over various intervals (1-24 h). All DD recordings were started after at least $7 \mathrm{~d}$ of constant conditions. The mean duration of individual bouts of vigilance states was analyzed for the 12-h light/subjective day and 12-h dark/subjective night periods, and between ZT6 and ZT12 on baseline day and following $6 \mathrm{~h}$ of SD. The total amount of NREMS during SD was calculated as well as the latency to the first $>25$ epochs (100 s) of NREMS after $6 \mathrm{~h}$ of SD. Two mice from the $\mathrm{SCN}^{\mathrm{Con}}$ group were excluded from the sleep analysis ( $n=1 \mathrm{LD}$ and DD; and $n=1$ from DD only) because of the lack of sleepwake data. Spectral analysis was computed for consecutive 4-s epochs by a fast Fourier transform (frequency range: $0.5-49.80 \mathrm{~Hz}$; resolution $0.24 \mathrm{~Hz}$; Hanning window function) on the EEG signal for wakefulness,
NREMS and REMS. Genotypic differences were determined in DD over a complete circadian cycle, and expressed as either absolute EEG power or a percentage of total EEG power (i.e., relative EEG power) within all vigilance states for each mouse. Epochs containing EEG artefacts were discarded from the analyses. The time course of EEG $\delta$ activity $(1-4 \mathrm{~Hz})$ during NREMS was also computed in 2-h bins during LD and DD and after 6-h SD and presented as absolute EEG power and/or relative (i.e., as a percentage of the mean) to 24 -h baseline for each mouse.

Sleep deprivation and novel object recognition (NOR) test

Mice were recorded for a $24-\mathrm{h}$ baseline day followed by $6-\mathrm{h}$ SD and a further 18-h recovery sleep. SD (ZT0-ZT6) involved gentle procedures, i.e., introduction of novel objects such as nesting material, "fun tubes" and an initial cage change. NOR was tested in dim red light ( $<10$ lux) between ZT20 and ZT22, in a red Perspex box measuring $50 \times 50 \times 50$ $\mathrm{cm}$ with an overhead camera (Logitech Carl Zeiss Tessar HD 1080P) placed above the arena. The mice were habituated to the arena without objects for $10 \mathrm{~min}$, followed by an initial familiarization session $24 \mathrm{~h}$ later where they were exposed to two identical objects for $10 \mathrm{~min}$ (plain or patterned Perspex objects, e.g., square, pyramid, oval, egg-cup all of similar sizes). After $24 \mathrm{~h}$, the mice were re-tested with one of the objects being replaced by a novel object of similar size. Animals are assumed to have remembered the familiar object if they spend significantly more time investigating the novel object during the test phase. Investigation was considered when the mouse nose-pointed within $1 \mathrm{~cm}$ of the objects, but was not included if the mouse was climbing on the objects. The discrimination index (DI) was calculated as the difference between the time spent exploring the novel and familiar objects divided by the total time 
spent exploring the two objects $\left[\mathrm{DI}=\left(\mathrm{T}_{\mathrm{N}}-\mathrm{T}_{\mathrm{F}}\right) /\left(\mathrm{T}_{\mathrm{N}}+\mathrm{T}_{\mathrm{F}}\right)\right]$. The time animals spent exploring each object in both the familiarization and test sessions were analyzed offline from the video recordings, using software designed by the laboratory of Prof W. Wisden, Imperial College, London, United Kingdom (Yu et al., 2014) with the experimenter blind to the genotype of the animal.

\section{Experimental design and statistical analysis}

Analyses were conducted in Prism version 9.1.2 for macOS X (GraphPad software). One-way or two-way ANOVA, with repeated measures (RM) where relevant, and with post hoc Tukey's, Dunnett's, or Sidak's multiple comparisons tests were used to compare changes in sleep/wake parameters across genotypes. Where sphericity of the data was not assumed a Geisser-Greenhouse's $\varepsilon$ correction was used (and so fractional degrees of freedom values are used to compute a $p$ value), as recommended in Prism. Data from the running-wheels were analyzed using ClockLab (Actimetrics Inc.), running within MATLAB (MathWorks). Circadian period $\left(\chi^{2}\right.$ periodogram analysis; unpaired Student's $t$ test between WT and $\mathrm{SCN}^{\mathrm{Cryl}}$ mice) and mean DD activity profiles were calculated for each animal, where activity was averaged over $8-10 \mathrm{~d}$ of activity and organized into 0.1 -h bins. Comparisons of sleep-wake bouts, duration and frequency were made using ANOVA between genotypes and within genotype. In all cases, the experimental unit was an individual mouse. Male mice were used to avoid the confounding effect of oestrus cycles on circadian behavior patterns observed in female mice. Following attrition because of technical difficulties, the three treatment-group sizes were WT $n=5, \mathrm{SCN}^{\mathrm{Con}} n=7$, and SCN ${ }^{\mathrm{Cry} 1}$ $n=7$. Given the variance of our measures, these sample sizes would yield a statistical power of $90-95 \%$ ( $\mathrm{G}^{\star}$ Power 3.1, University of Dusseldorf, Dusseldorf, Germany).

\section{Results}

\section{Local expression of Cry1 in the suprachiasmatic} hypothalamus initiates circadian wheel-running behavior in clockless mice

Local, bilateral AAV-mediated nuclear expression of Cry1::EGFP in the suprachiasmatic hypothalamus of Cry1/Cry2-null mice was evident from the EGFP tag (Fig. $1 B-D$ ). It was limited to the SCN and the immediately surrounding hypothalamus, in some cases extending into the anterior hypothalamic area and paraventricular nucleus (PVN) and/or posteriorly to the retrochiasmatic area and/or anteriorly to the medial preoptic area (Fig. $1 E$ ). Cryl did not extend into the dorsal or lateral hypothalamus, and there was no consistent pattern of extra-SCN expression between animals: the SCN was the only target common to all mice (see overlay in Fig. $1 E$ ). The total proportion of transfected cells within the paired SCN, together, was $46.5 \pm 6.5 \% \quad(n=7$; range $24-74 \%$ ), as assessed by the ratio of EGFP-positive cells to DAPI-positive cells using ImageJ, which is in agreement with our previous studies (Maywood et al., 2018; Brancaccio et al., 2019). WT mice exhibited robust circadian cycles of wheel-running behavior, whereas Cry1,2-null mice targeted with EGFP $\left(\mathrm{SCN}^{\mathrm{Con}}\right)$ were arrhythmic in DD before and after surgery (Fig. $2 A)$. In contrast, previously arrhythmic $\mathrm{SCN}^{\mathrm{Cry} 1}$ mice exhibited robust circadian wheel-running behavior following expression of Cryl::EGFP, as demonstrated previously (Maywood et al., 2018). The mean postsurgery activity profile between groups shows the significant circadian rhythmicity in both the WT and SCN ${ }^{\text {Cryl }}$ mice, whereas activity in $\mathrm{SCN}^{\mathrm{Con}}$ mice was distributed evenly across the circadian cycle, phase-referenced to the prior LD cycle (Fig. $2 B$ ). Furthermore, the period of $24.8 \pm 0.2 \mathrm{~h}(n=7$; Fig. $2 C)$ in $\mathrm{SCN}^{\mathrm{Cry} 1}$ mice was significantly longer than in WT $(n=5$; $24.1 \pm 0.1 \mathrm{~h}$ ) and diagnostic of a Cryl-driven TTFL (van der Horst et al., 1999; Maywood et al., 2018). Finally, non-parametric analysis of locomotor activity confirmed the excellent circadian organization of WT mice, and its disorganization in $\mathrm{SCN}^{\mathrm{Con}}$ mice, which had low relative amplitude and stability and high variability (Fig. 2D-F). In contrast, previously arrhythmic $\mathrm{SCN}^{\mathrm{Cry} 1}$ mice showed robust circadian behavior after surgery, with significantly improved organization comparable to WT mice, and a non-significant trend for lower intradaily variability (see legend to Fig. 2 for statistical analyses).

Rescue of circadian sleep/wake patterning in $\mathrm{SCN}^{\mathrm{Cry} 1}$ mice Initiation of circadian control of wheel-running behavior by AAV-mediated genetic complementation made it possible to explore the degree of control to sleep mediated by molecular competence in the suprachiasmatic hypothalamus. EEG spectral analysis showed that under DD, in the absence of any masking or other effects of light, the different vigilance states exhibited their characteristic neurophysiological features (Fig. 3). Moreover, the genotype had no significant effect on these parameters, confirming that the absence of Cry proteins does not affect the core molecular and neural machinery that generates the states of wakefulness, REMS, and NREMS

The total amount of wake did not vary between groups under LD $(24 \mathrm{~h}$ ) or DD (circadian cycle; Fig. $4 A, B$; wake LD: 1 $\times$ ANOVA $F_{(2,16)}=1.615, p=0.2298$; wake DD: $1 \times$ ANOVA $\left.F_{(2,15)}=3.26, p=0.0669\right)$, but compared with WT controls, both $\mathrm{SCN}^{\mathrm{Con}}$ and $\mathrm{SCN}^{\mathrm{Cry} 1}$ mice exhibited more NREMS under LD, as reported (Wisor et al., 2002; NREMS LD: $1 \times \operatorname{ANOVA~} F_{(2,16)}=$ 4.7, $p=0.024$; Tukey's multiple comparisons test: WT vs SCN ${ }^{\text {Con }}$ $p=0.0347$, WT vs SCN ${ }^{\text {Cry1 }} p=0.0397, \mathrm{SCN}^{\text {Con }}$ vs $\mathrm{SCN}^{\mathrm{Cry} 1}$ $p=0.9969$; NREMS DD: $1 \times$ ANOVA $\left.F_{(2,15)}=2.2, p=0.15\right)$. In $\mathrm{DD}$, but not $\mathrm{LD}$, both $\mathrm{SCN}^{\mathrm{Con}}$ and $\mathrm{SCN}^{\mathrm{Cry} 1}$ mice showed a small elevation in REMS (REMS LD: $1 \times \operatorname{ANOVA} F_{(2,16)}=2.9, p=0.08$; REMS DD: $1 \times$ ANOVA $F_{(2,15)}=5.7, p=0.015$; Tukey's multiple comparisons test: WT vs SCN ${ }^{\mathrm{Con}} p=0.0245$, WT vs SCN ${ }^{\text {Cry } 1}$ $p=0.0232, \mathrm{SCN}^{\mathrm{Con}}$ vs $\mathrm{SCN}^{\mathrm{Cry} 1} p=0.9967$; Fig. $\left.4 C-F\right)$. These likely represent Cry-dependent traits independent of the SCN.

We then examined the temporal distribution of sleep/wake. Under LD, WT mice showed appropriate nocturnal wakefulness and more NREMS and REMS in daytime (Fig. 4A,C,E). Equally, both Cry1, Cry2-null groups, $\mathrm{SCN}^{\mathrm{Con}}$ and $\mathrm{SCN}^{\mathrm{Cry} 1}$, had clearly defined light/dark differences in wake and NREMS (Fig. 4A,C), whereas $\mathrm{SCN}^{\mathrm{Con}}$ mice did not show significant light/dark differences in REMS in LD (Fig. 4E). Despite the light/dark organization of the sleep/wake patterns, both the $\mathrm{SCN}^{\mathrm{Con}}$ and $\mathrm{SCN}^{\mathrm{Cry} 1}$ mice spent significantly less time in wake in the dark phase compared with WT (Fig. 4A,C,E; as assessed by post hoc Sidak's and Tukey's multiple comparisons tests within genotype and between genotypes, respectively, where there was a significant interaction and/or genotype effect in a $2 \times$ RMANOVA; see legend to Fig. 4 for details).

Under DD, WT mice again showed clear differences between subjective day (rest phase; higher levels of NREMS and REMS) and subjective night (active phase; more wake; Fig. $4 B, D, F)$. In contrast, under $\mathrm{DD}$, the $\mathrm{SCN}^{\mathrm{Con}}$ mice showed no significant circadian patterning to vigilance states (Fig. 4B,D,F; no significant rest/active differences within genotype assessed using $2 \times$ RMANOVA with post hoc Sidak's multiple comparisons tests; see legend to Fig. 4 for statistical analyses). Unlike in LD, where the light imposed a level of organization on the sleep/wake profiles, under DD the $\mathrm{SCN}^{\mathrm{Con}}$ mice spent significantly more time in wake (Fig. $4 B$ ) and less time in NREMS in the circadian day (Fig. 4D), and significantly less time in wake and more NREMS and REMS in circadian night, compared with WT (Fig. $4 B, D, F$; as 
A
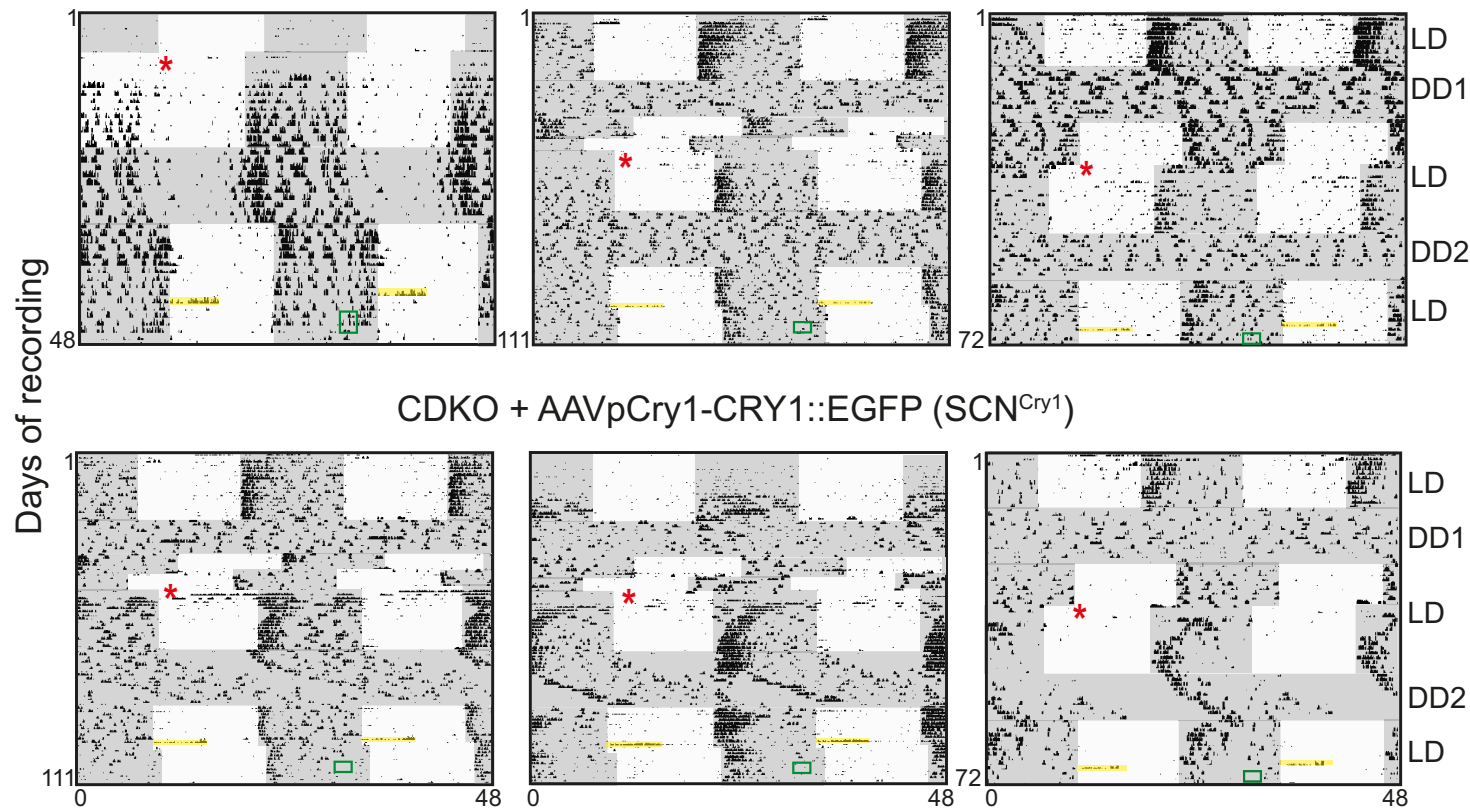

B

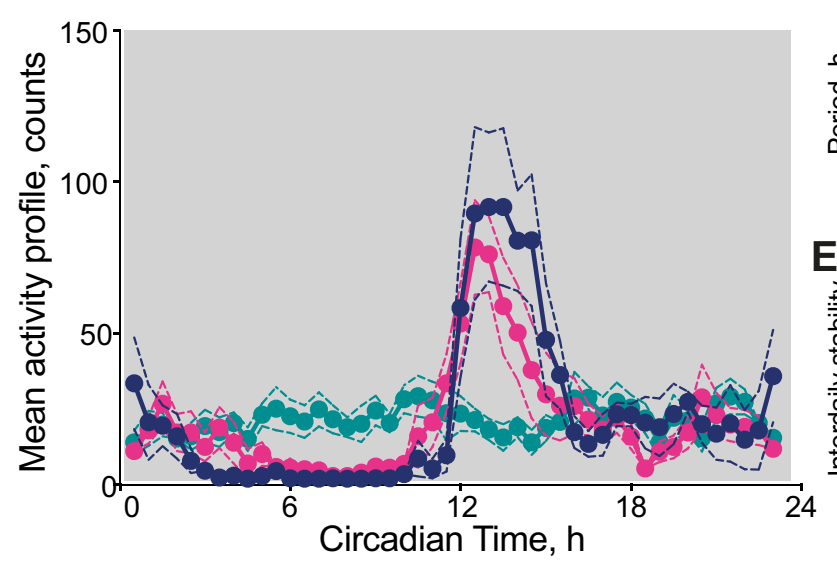

C

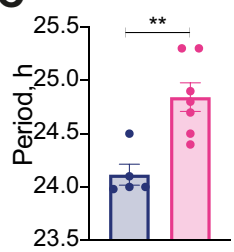

E

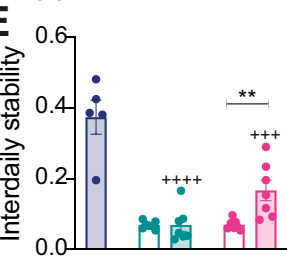

D

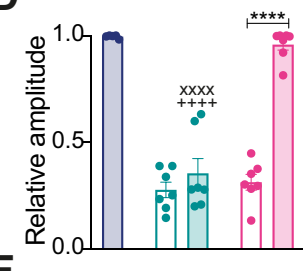

$\mathbf{F}$

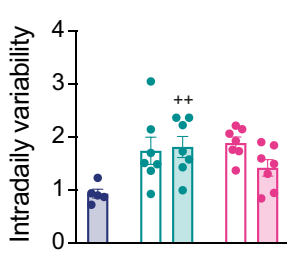

Figure 2. Local expression of Cry1 in the suprachiasmatic hypothalamus initiates circadian wheel-running behavior in clockless mice. A, Double-plotted wheel-running traces from a WT (top left), two $\mathrm{SCN}{ }^{\mathrm{Con}}$ (middle and top right), and three representative traces from $\mathrm{SCN}^{\mathrm{Cry} 1}$ mice (bottom panel). Gray shaded areas represent darkness (LD; DD) before (DD1) and after (DD2) surgery (denoted by the red asterisk). The $\mathrm{CDKO}$ mice were arrhythmic presurgery, and the $\mathrm{SCN}^{\mathrm{Con}}$ mice remained arrhythmic postsurgery, whereas $\mathrm{SCN}^{\mathrm{Con}}$ mice showed significant circadian rhythmicity postsurgery. The yellow bar highlights the 6 -h SD and the green box the timing of the NOR testing. $\boldsymbol{B}$, Mean activity ( \pm SEM) counts over a circadian cycle in WT ( $n=5$; blue), SCN ${ }^{\text {Con }}$ ( $n=7$; green), and $S C C^{C r y 1}$ ( $n=7$; magenta); $2 \times$ RMANOVA: interaction: $F_{(90,630)}=4.0, p<0.0001$; genotype: $F_{(2,14)}=0.09, p=0.9138 ;$ time: $\left.F_{(45,630)}=10.77, p<0.0001\right)$. C $-F$, Mean $( \pm S E M)$ and individual values of period $(\boldsymbol{C})$, relative amplitude $(\boldsymbol{D})$, interdaily stability $(\boldsymbol{E})$, and intradaily variability $(\boldsymbol{F})$ in WT $\left(n=5 ;\right.$ blue), $\mathrm{SCN}^{\mathrm{Con}}\left(n=6\right.$; green), and $\mathrm{SCN}^{\mathrm{Cry1}}(n=7$; magenta). 0pen bars are presurgery, shaded bars postsurgery (period: two-tailed unpaired Student's $t$ test: $t_{(10)}=4.03 ; p<0.005 ; 2 \times$ RMANOVA comparison of presurgery and postsurgery measures between $\mathrm{SCN}^{\mathrm{Con}}$ and SCN ${ }^{\mathrm{Cry} 1}$ genotypes: relative amplitude: interaction: $F_{(1,12)}=29.6 p=0.0002$, genotype: $F_{(1,12)}=70.35 p<0.0001$; surgery: $F_{(1,12)}=56.17 p<0.0001$; interdaily stability: interaction: $F_{(1,12)}=7.0 p=0.021$, genotype: $F_{(1,12)}=6.4 p=0.0265$; surgery: $F_{(1,12)}=8.19 p=0.0143$; intradaily variability: interaction: $F_{(1,12)}=2.15 p=0.17$, genotype: $F_{(1,12)}=0.036 p=0.852$; surgery: $F_{(1,12)}=0.36 p=0.5586 ; 1 \times$ ANOVA comparing WT vs postsurgery of $S \mathrm{SN}^{\mathrm{Con}}$ and $S \mathrm{SN}^{\mathrm{Cry} 1}$ genotype: relative amplitude: $F_{(2,16)}=59.9 p<0.0001$, post hoc Tukey's multiple comparisons tests WT vs $S_{C N}{ }^{C o n} p<0.0001$, WT vs $S C N^{C r y 1} p=0.8419, S_{C N}{ }^{C r y 1}$ vs $S C N^{C o n} p<0.0001$; interdaily stability: $F_{(2,16)}=23.15 p<0.0001$, post hoc Tukey's multiple comparisons tests WT

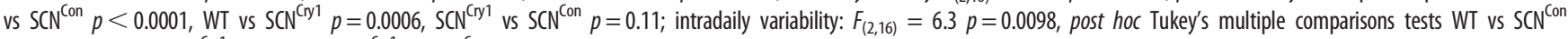
$p=0.0072$, WT vs $\operatorname{SCN}^{\mathrm{Cry} 1} p=0.1247$, $\mathrm{SCN}^{\mathrm{Cry} 1}$ vs $\mathrm{SCN}^{\mathrm{Con}} p=0.2765 ;{ }^{* *} p<0.01,{ }^{* * *} p<0.0001 ;++p<0.0001,+++p<0.001,++++p<0.01$ vs WT; $\operatorname{xxxxp}^{*}<0.0001$ vs $\left.\mathrm{SCN}{ }^{\mathrm{Cr} 1}\right)$.

assessed by post hoc Tukey's multiple comparisons between groups where there was a significant interaction and/or genotype effect in a $2 \times$ RMANOVA; see legend to Fig. 4 for details). In contrast, $\mathrm{SCN}{ }^{\mathrm{Cry} 1}$ mice showed robust circadian organization to the sleep-wake cycle, with clear subjective day and night differences comparable to those of WT controls across all vigilance states.
Nevertheless, these mice did spend significantly less time in wake and more time in NREMS and REMS during the circadian night compared with WT (Fig. 4B,D,F; as assessed by post hoc Tukey's multiple comparisons between genotypes where there was a significant interaction and/or genotype effect in a $2 \times$ RMANOVA; see legend to Fig. 4 for details). The loss of circadian organization to 
A

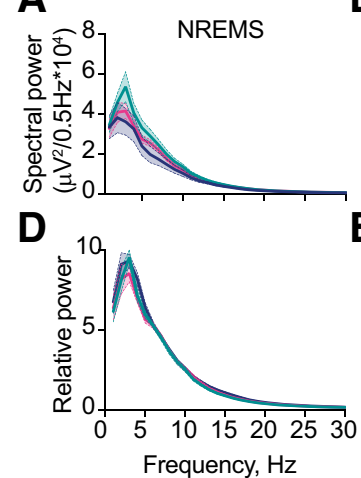

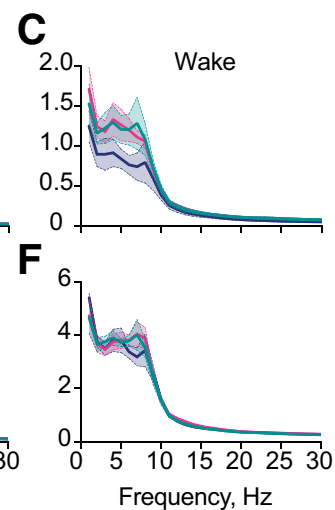

Figure 3. Local expression of Cry1 in the suprachiasmatic hypothalamus organizes circadian sleep/wake patterning. $\boldsymbol{A}-\boldsymbol{C}$, Mean baseline raw EEG spectral power in $\operatorname{NREMS}(\boldsymbol{A})$, REMS $(\boldsymbol{B})$, and wake $(\boldsymbol{C})$ over a circadian cycle (DD; $2-$ $\times$ RMANOVA: NREMS: interaction: $F_{(120,900)}=1.0, p=0.43$, genotype: $F_{(2,15)}=1.12, p=0.3555$, frequency: $F_{(1.6,23.4)}=$ 143.2, $p<0.0001$; REMS: interaction: $F_{(120,900)}=1.02$, genotype: $F_{(2,15)}=1.25, p=0.3151$, frequency: $F_{(1.9,25.9)}=$ 98.69, $p<0.0001 p=0.44$; wake: interaction: $F_{(120,900)}=0.68, p>0.99$, genotype: $F_{(2,15)}=1.52, p=0.2506$, frequency: $\left.F_{(1.6,23.4)}=55.74, p<0.0001\right)$. $\boldsymbol{D}-\boldsymbol{F}$, Mean baseline relative EEG spectral power (relative to total power) in NREMS $(\boldsymbol{D})$, REMS $(\boldsymbol{E})$, and wake $(\boldsymbol{F})$ over a circadian cycle (DD; $2 \times$ RMANOVA: NREMS: interaction: $F_{(120,900)}=0.52$, $p>0.99$, genotype: $F_{(2,15)}=1.28, p=0.3064$, frequency: $F_{(1.6,26.1)}=368.1, p<0.0001$; REMS: interaction: $F_{(120,900)}=$ 0.91, $p=0.7926$, genotype: $F_{(2,15)}=0.4, p=0.6768$, frequency: $F_{(2.3,31.9)}=214.0, p<0.0001$; wake: interaction: $F_{(120,900)}=0.32, p>0.99$, genotype: $F_{(2,15)}=0.60, p=0.5615$, frequency: $\left.F_{(1.6,24.7)}=160.5, p<0.0001\right)$. WT $(n=5)$, blue; $\operatorname{SCN}^{\mathrm{Con}}(n=6)$, green; $\mathrm{SCN}^{\mathrm{Cry} 1}(n=7)$, magenta.

sleep in $\mathrm{SCN}^{\mathrm{Con}}$ mice was therefore reversed in $\mathrm{SCN}^{\text {Cry1 }}$ mice, enabling the de novo establishment of a more WT-like organization to the diurnal/circadian patterning to sleep/wake.

A finer-grained, 2-h resolution, analysis of the 24-h distribution of sleep/wake emphasized further the effects on sleep-wake patterning of global Cryl,2 deficiency and local restoration of clock function in the SCN region (Fig. 5; as assessed by post hoc Tukey's multiple comparisons tests between genotypes where there was either a significant interaction and/or genotype effect in a $2 \times$ RMANOVA; see legend to Fig. 5 for details). Under LD, all groups exhibited a daily pattern of vigilance states, but with a reduced amplitude in the $\mathrm{SCN}^{\mathrm{Con}}$ mice, likely reflecting their poor behavioral entrainment to, and/or masking by, the photoschedule (Fig. 5A-D). Furthermore, in the second half of the light phase (ZT6-ZT12), $\mathrm{SCN}^{\mathrm{Con}}$ mice had significantly more wakefulness (+50 min) and less NREMS ( $-40 \mathrm{~min}$ ) compared with WT mice. Expression of Cry1 in the SCN region reversed these deficits (1$\times$ ANOVA ZT6-ZT12: wake amount: $F_{(2,16)}=8.3, p<0.005$; post hoc Tukey's multiple comparison test: WT vs $\mathrm{SCN}^{\mathrm{Con}} p=0.003$; WT vs $\mathrm{SCN}^{\mathrm{Cry1}} p=0.36$; $\mathrm{SCN}^{\text {Cry1 }}$ vs $\mathrm{SCN}^{\mathrm{Con}} p=0.035$; NREMS amount: $F_{(2,16)}=7.2, p=0.0059$; post hoc Tukey's multiple comparison test: WT vs $\mathrm{SCN}^{\mathrm{Con}} p=0.007$; WT vs $\mathrm{SCN}^{\text {Cry1 }} p=0.55$; $\mathrm{SCN}^{\mathrm{Cry1}}$ vs $\mathrm{SCN}^{\mathrm{Con}} p=0.036$ ). Conversely, at the end of the dark phase, WT mice showed more wake and less NREMS and REMS than both CDKO groups (Fig. 5A-C). Finally, the amount of REM as a proportion of total sleep (TS = NREMS + REMS) was high in day and low at night in WT mice and this pattern was replicated by $\mathrm{SCN}^{\mathrm{Con}}$ and $\mathrm{SCN}^{\mathrm{Cry} 1}$ (Fig. $5 D$ ). Under LD, therefore, loss of Cry proteins altered the temporal distribution of vigilance states across the $24 \mathrm{~h}$, and this was partially restored by Cryl expression focused on the SCN.

The group differences between $\mathrm{WT}$ and $\mathrm{SCN}^{\mathrm{Con}}$ mice were amplified in DD (Fig. 5E-H; see legend to Fig. 5 for 2$\times$ RMANOVA analyses between genotypes, although not possible to do statistics including the group $\mathrm{SCN}^{\mathrm{Cry} 1}$ as these mice have a different endogenous free-running period and so a different timescale). Whereas WT exhibited robust circadian patterning, $\mathrm{SCN}^{\mathrm{Con}}$ mice failed to show any significant organization of wake, NREMS or REMS across the circadian cycle (Fig. 5E-G). Similarly, in WT mice the amount of REMS as a proportion of TS, which under DD is a measure of circadian control independent of changes in the absolute amount of wakefulness, was highly circadian, whereas it was not in $\mathrm{SCN}^{\mathrm{Con}}$ mice $\left(\right.$ REM/TS: WT: $1 \times$ ANOVA $F_{(11,48)}=5.8$, $p<0.0001 ; \mathrm{SCN}^{\mathrm{Con}}: F_{(11,60)}=0.9$; Fig. $\left.5 H\right)$. Expression of Cryl had a marked restorative effect on sleep/wake patterns in DD. The $\mathrm{SCN}^{\mathrm{Cry1}}$ mice showed a more WT-like organization of the sleep/wake cycle across circadian day and night (Fig. $5 E-G$ ), although this rescue was not complete as they did have slightly less wakefulness $(\sim 12 \%)$ and more NREMS $(\sim 10 \%)$ and REMS $(\sim 2 \%)$ in the circadian night (CT12-CT24) compared with WT. Levels of REMS/TS were higher overall in $\mathrm{SCN}^{\mathrm{Cry} 1}$ mice, but they nevertheless showed a significant circadian rhythm in the distribution of REMS/TS over the circadian cycle (REMS/TS: SCN ${ }^{\text {Cry1 }}$ : $1 \times$ ANOVA $F_{(12,78)}=7.72, p<0.0001$; Fig. $\left.5 H\right)$ and, as with the WT mice but not the $\mathrm{SCN}^{\mathrm{Con}}$ mice, had significantly lower overall levels of REMS/TS in circadian night (total CT12-CT24) than in circadian day (total CT0-CT12; total REMS/TS in circadian day/circadian night: $2 \times$ RMANOVA: interaction: $F_{(2,15)}=21.12$, $p<0.0001$, genotype: $F_{(2,15)}=5.18, p=0.0195$; time: $F_{(1,15)}=$ $31.94, p<0.0001$; post hoc Sidak's multiple comparison test: total in circadian day vs total in circadian night: WT $p=0.0056$; $\left.\mathrm{SCN}^{\mathrm{Con}} p=0.38 ; \mathrm{SCN}^{\mathrm{Cry1}} p<0.0001\right)$. Together, the LD and DD analyses confirm the interaction between light and the circadian system in organizing sleep/wake cycles (Tsai et al., 2009), and suggest that the suprachiasmatic clockwork has a sleep-promoting/wake-suppressing effect in the second half of the light/rest phase of the LD and DD cycles (ZT/CT6-CT12). Nevertheless, in the absence of a lighting cycle, a molecularly competent SCN is able to impose a circadian distribution of sleep-wake patterns in an otherwise clockless mouse.

\section{Consolidation of disrupted sleep/wake architecture in SCN ${ }^{\text {Cryl }}$ mice}

Loss of circadian patterning to sleep-wake in $\mathrm{SCN}^{\mathrm{Con}}$ mice and its restoration to WT-like organization in $\mathrm{SCN}^{\mathrm{Cry} 1}$ mice were indicators of the autonomous power of the suprachiasmatic clock. We then examined its effect on sleep/wake architecture, as there was no a priori reason to expect that a functional SCN could reinstate a WT-like structure. When entrained to an LD cycle, WT mice showed a longer duration of wake in the dark (active) phase, and correspondingly fewer episodes of NREMs and REMS at night and more in the light (rest) phase (with no systematic changes in their duration; Fig. $6 A, B$; as assessed by post hoc Sidak's and Tukey's multiple comparisons tests within genotype and between genotypes, respectively, where there was a significant interaction and/or genotype effect in a 2$\times$ RMANOVA; see legend to Fig. 6 for details). In comparison, $\mathrm{SCN}^{\mathrm{Con}}$ mice showed weaker consolidation. They did not exhibit longer wake bouts during the dark (active) phase than during the light (rest) phase in LD, with nocturnal wake bouts being shorter than in WT mice, and NREMS bouts longer in both the light and dark phases, as reported previously for Cry1, Cry2-null mice 
(Wisor et al., 2002). Nevertheless, in $\mathrm{SCN}^{\mathrm{Con}}$ mice, although bouts of NREMS and REMS were more frequent in the light compared with the dark phase, they had significantly fewer bouts during the daytime (rest phase) than WT mice (Fig. 6B; as assessed by post hoc Tukey's multiple comparisons between genotypes where there was a significant interaction and/or genotype effect in a $2 \times$ RMANOVA; see legend to Fig. 6 for details). Local expression of Cryl in the SCN region reversed the deficits of $\mathrm{SCN}^{\mathrm{Con}}$ mice in LD: the duration of wake bouts was significantly longer at night compared with $\mathrm{SCN}^{\mathrm{Con}}$ mice, and no different from WT measures, and the duration and number of NREMS and REMS bouts was comparable to WT mice (Fig. 6A,B; as assessed by post hoc Tukey's multiple comparisons between genotypes where there was a significant interaction and/or genotype effect in a $2 \times$ RMANOVA; see legend to Fig. 6 for details).

The differences between groups in sleep consolidation were even more stark under circadian free-running conditions. WT mice retained their longer duration of nocturnal wake bouts (active phase) and more bouts of NREMS and REMS in circadian daytime (rest phase; Fig. $6 C, D ; 2 \times$ RMANOVA with post hoc Sidak's multiple comparisons tests within genotype; see legend to Fig. 6 for statistical analyses). SCN ${ }^{\mathrm{Con}}$ mice, however, exhibited no significant rest/ active differences in the duration or number for any vigilance state. The durations of wake bouts in circadian night were significantly shorter than in WT mice, and the number of nocturnal bouts of all three states were more numerous than in WT mice, reflecting the loss of consolidated wake in circadian night. Equally, $\mathrm{SCN}^{\mathrm{Con}}$ exhibited significantly more episodes of wake in circadian daytime than did WT mice (as assessed by post hoc Sidak's and Tukey's multiple comparisons tests within genotype and between genotypes, respectively, where there was a significant interaction and/or genotype effect in a 2$\times$ RMANOVA; see legend to Fig. 6 for details). Importantly, all of these deficiencies in $\mathrm{SCN}^{\mathrm{Con}}$ mice were reversed by expression of Cryl in the SCN and adjacent tissue. In $\mathrm{SCN}^{\mathrm{Cry} 1}$ mice, nocturnal wake bout duration was significantly longer than in subjective day, albeit not as long as in WT mice, and bouts of NREMS and REMS were significantly more numerous in circadian day
12L:12D

A
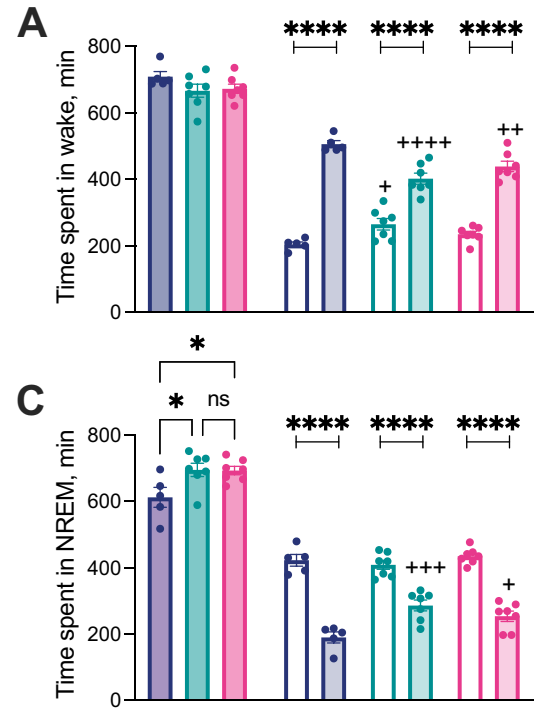

$E$

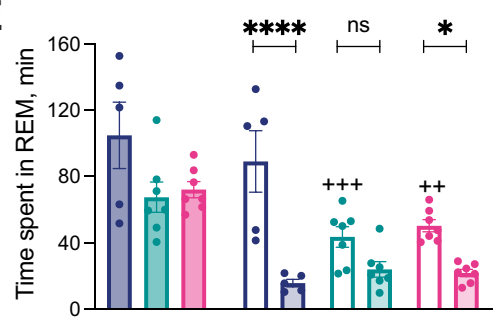

DD

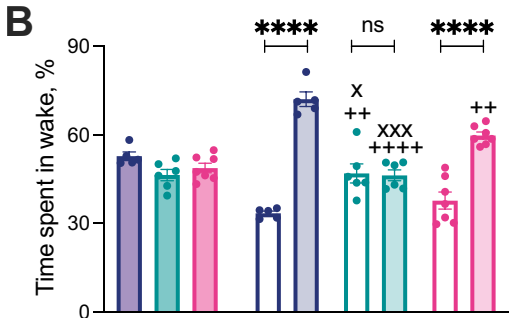

D
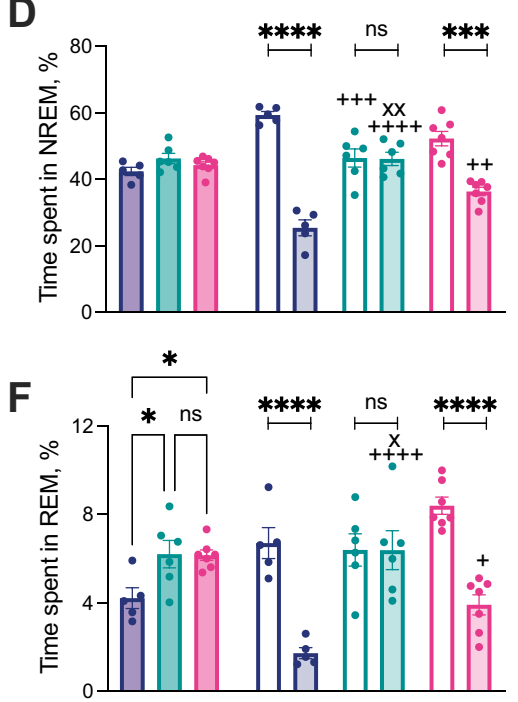

Figure 4. Local expression of $\mathrm{Cry} 1$ in the suprachiasmatic hypothalamus organizes circadian sleep/wake patterning. $A, C, E$, Time spent in wakefulness $(\boldsymbol{A})$, NREMS $(\boldsymbol{C})$, and REMS $(\boldsymbol{E})$ in 12hL:12D (minutes; mean \pm SEM and individual points). Dark shaded bars on the left-hand side (LHS) show time over $24 \mathrm{~h}$ (see Results; 1×ANOVA: NREMS LD $24 \mathrm{~h}$, post hoc Tukey's multiple comparisons test $+p<0.05$ vs WT), with the 12-h light (clear) and 12-h dark (lightly shaded) bars on the right-hand side (RHS). There is clear day (rest)/night (active) organization in all vigilance states in both WT and $\mathrm{SCN}^{\mathrm{Cry} 1}$ mice but only in wake and NREMS for the $S \mathrm{SN}^{\mathrm{Con}}$ mice $\left(2 \times\right.$ RMANOVA: wake: interaction: $F_{(2,16)}=11.94, p=0.0007$, genotype: $F_{(2,16)}=1.615$, $p=0.2297$; time: $F_{(1,16)}=256.4, p=0.0007$, post hoc Sidak's multiple comparisons test day-night: WT, $\operatorname{SCN}^{\mathrm{Con}}$, and SCN ${ }^{\mathrm{Cr} 1}$ all $p<0.0001$, post hoc Tukey's multiple comparisons test, day: WT vs $\mathrm{SCN}^{\mathrm{Con}} p=0.0174$, WT vs $\mathrm{SCN}^{\mathrm{Cry} 1} p=0.3224$, $\mathrm{SCN}^{\mathrm{Con}}$ vs $\mathrm{SCN}^{\mathrm{Cry} 1} p=0.2648$, night: WT vs $\mathrm{SCN}^{\mathrm{Con}} p<0.0001$, WT vs $\mathrm{SCN}^{\mathrm{Cry} 1} p=0.0085$, $\mathrm{SCN}^{\mathrm{Con}}$ vs $\mathrm{SCN}^{\mathrm{Cr} 1} p=0.1521$; NREMS: interaction: $F_{(2,16)}=6.5, p=0.0085$, genotype: $F_{(2,16)}=4.144, p=0.0355$; time: $F_{(1,16)}=214.1, p<0.000$, post hoc Sidak's multiple comparisons test day-night: WT, $S C N^{C o n}$, and $S C N^{\text {Cry } 1}$ all $p<0.0001$, post hoc Tukey's multiple comparisons test, day: WT vs $S C N^{\text {Con }}$ $p=0.8075$, WT vs SCN ${ }^{\mathrm{Cr} 1} p=0.8383, \mathrm{SCN}^{\mathrm{Con}}$ vs $\mathrm{SCN}^{\mathrm{Cry1}} p=0.4021$, night: WT vs $\mathrm{SCN}^{\mathrm{Con}} p=0.0003$, WT vs $\operatorname{SCN}^{\mathrm{Cry} 1} p=0.017$, $S C N^{\text {Con }}$ vs $S C N^{\text {Cry1 }} p=0.2505$; REMS: interaction: $F_{(2,16)}=9.1, p=0.0023$, genotype: $F_{(2,16)}=0.0821, p=0.0821$; time: $F_{(1,16)}=$ $59.79, p<0.0001$, post hoc Sidak's multiple comparisons test day-night: WT and $\operatorname{SCN}^{\mathrm{Cry} 1} p<0.0001$, $\operatorname{SCN}^{\mathrm{Con}} p=0.1026$, post hoc Tukey's multiple comparisons test, day: WT vs $\mathrm{SCN}^{\mathrm{Con}} p=0.0174$, WT vs $\mathrm{SCN}^{\mathrm{Cry} 1} p=0.3224, \mathrm{SCN}^{\mathrm{Con}}$ vs $\mathrm{SCN}^{\mathrm{Cry} 1} p=0.2648$, night: WT vs $S C N^{C o n} p<0.0001$, WT vs $S C N^{C r y 1} p=0.0085$, $S_{C N}{ }^{C o n}$ vs $\left.S C N^{C r y 1} p=0.1521\right) . B, D, F$, Time spent in wakefulness $(\boldsymbol{B})$, NREMS $(\boldsymbol{D})$, and REMS $(\boldsymbol{E})$ in DD (\% time; mean \pm SEM and individual points). Dark shaded bars on LHS show time over a circadian cycle ( $1 \times$ ANOVA: REMS DD, post hoc Tukey's multiple comparisons test $+p<0.05$ vs WT), with the circadian day/rest phase (clear) and circadian night/active phase (lightly shaded) bars on the RHS. There is clear circadian day (rest)/night (active) organization in all vigilance states in both WT and $\mathrm{SCN}^{\mathrm{Cry} 1}$ mice but only in the $\mathrm{SCN}^{\mathrm{Con}}$ mice (2×RMANOVA: wake: interaction: $F_{(2,15)}=35.74, p<0.0001$, genotype: $F_{(2,15)}=3.106, p=0.0744$; time: $F_{(1,15)}=117.2, p<0.0001$, post hoc Sidak's multiple comparisons test day-night: WT and $\mathrm{SCN}{ }^{\mathrm{C} r y 1} p<0.0001, \mathrm{SCN}^{\mathrm{Con}} p=0.9957$, post hoc Tukey's multiple comparisons test, day: WT

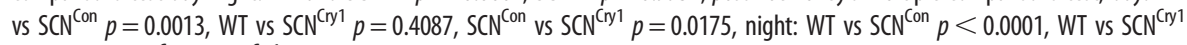
$p=0.0023$, SCN ${ }^{\mathrm{Con}}$ vs SCN ${ }^{\mathrm{Cr} 1} p=0.0005$; NREMS: interaction: $F_{(2,15)}=25.3, p<0.0001$, genotype: $F_{(2,15)}=2.196, p=0.1457$; time: $F_{(1,15)}=80.98, p<0.0001$, post hoc Sidak's multiple comparisons test day-night: WT $p<0.0001, \operatorname{SCN}^{\mathrm{Cr} y 1} p=0.0002$, $\mathrm{SCN}^{\mathrm{Con}} p=0.9996$, post hoc Tukey's multiple comparisons test, day: WT vs $\mathrm{SCN}^{\mathrm{Con}} p=0.0005$, WT vs $\mathrm{SCN}^{\mathrm{Cry} 1} p=0.0522$, $\mathrm{SCN}^{\mathrm{Con}}$ vs $S_{C N}{ }^{C r y 1} p=0.1075$, night: WT vs $S C N^{C o n} p<0.0001$, WT vs $S C N^{C r y 1} p=0.0021$, SCN ${ }^{C 0 n}$ vs SCN ${ }^{C r y 1} p=0.0036$; REMS: interaction: $F_{(2,15)}=19.67, p<0.0001$, genotype: $F_{(2,15)}=4.735, p=0.0255$; time: $F_{(1,15)}=78.41, p<0.0001$, post hoc Sidak's multiple comparisons test day-night: WT and $\mathrm{SCN}^{\mathrm{Cry1}} p<0.0001, \mathrm{SCN}^{\mathrm{Con}} p=0.9999$, post hoc Tukey's multiple comparisons test, day: WT vs $S C N^{\text {Con }} p=0.9361$, WT vs $S C \mathrm{CN}^{\mathrm{Cry} 1} p=0.1428 \mathrm{SCN}^{\mathrm{Con}}$ vs $\mathrm{SCN}^{\mathrm{Cr} y 1} p=0.0542$, night: WT vs $\mathrm{SCN}^{\mathrm{Con}} p<0.0001$, WT vs $\mathrm{SCN}^{\mathrm{Cr} 1} p=0.0432, \mathrm{SCN}^{\mathrm{Con}}$ vs $\left.\mathrm{SCN} \mathrm{N}^{\mathrm{Cr} 1} p=0.014\right) ;{ }^{*} p<0.05,{ }^{* * *} p<0.001$, ${ }^{* * *} p<0.0001$ within genotype; $+p<0.05$, $++p<0.01,+++p<0.001,++++p<0.0001$ versus $\mathrm{WT} ; \operatorname{xp}<0.05, \operatorname{xxp}<0.01, \operatorname{xxxp}<0.001$ versus $\mathrm{SCN}^{\mathrm{Cry} 1}$. WT $(n=5)$, blue; $\operatorname{SCN}^{\text {Con }}(n=6)$, green; $\operatorname{SCN}^{\mathrm{Cr} y 1}(n=7)$, magenta. 
A
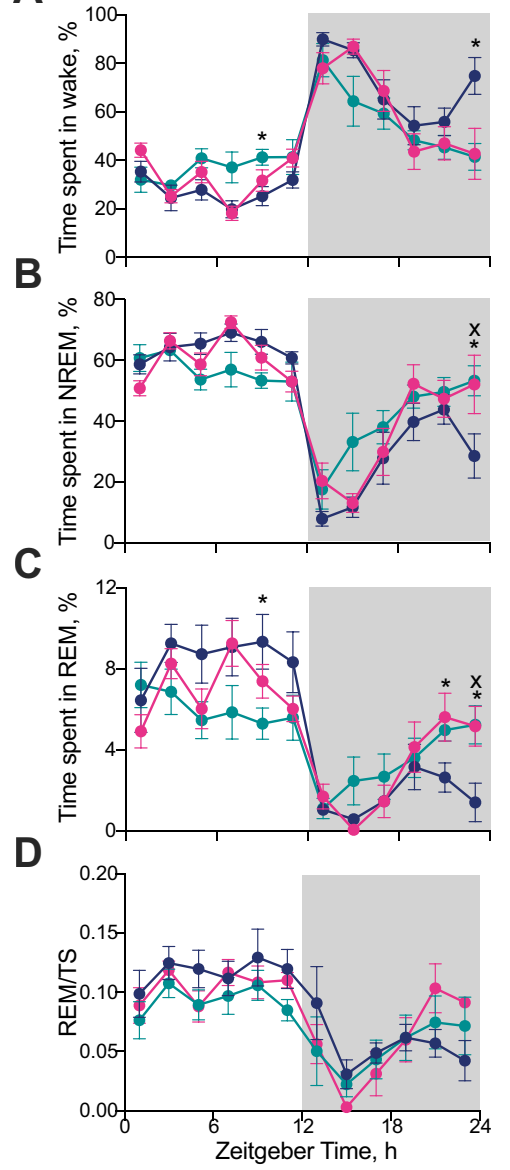

E

$F$

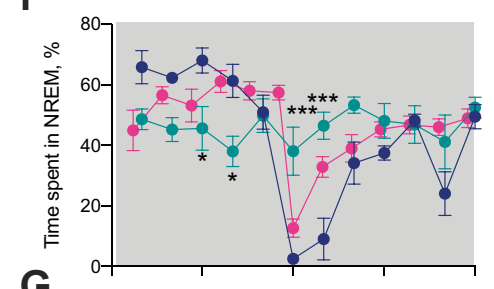

G

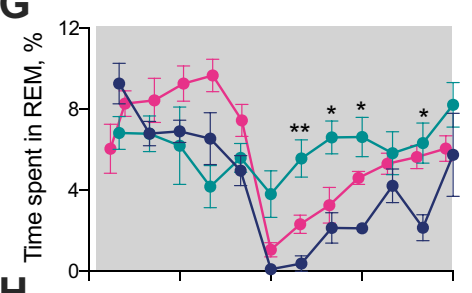

$\mathrm{H}$

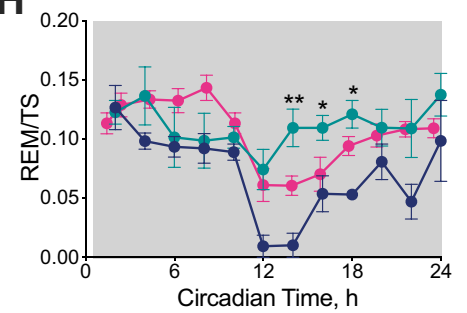

Figure 5. Local expression of Cry1 in the suprachiasmatic hypothalamus organizes circadian sleep/wake patterning. $\boldsymbol{A}-\boldsymbol{D}, 2$-h profiles (mean \pm SEM) of wakefulness $(\boldsymbol{A})$, NREMS $(\boldsymbol{B})$, REMS $(\boldsymbol{C})$, and REMS/TS $(\boldsymbol{D})$ in WT ( $n=5$; blue), $\operatorname{SCN}^{\mathrm{Con}}\left(n=7\right.$; green), and $\operatorname{SCN}^{\mathrm{Cry1}}$ ( $n=7$; magenta) under a 12/12 h light/dark photoschedule ( $2 \times$ RMANOVA wake: interaction: $F_{(22,176)}=2.23, p=0.0022$, genotype: $F_{(2,16)}=0.9038, p=0.4247$; time: $F_{(11,176)}=30.82, p<0.0001$; NREMS: interaction: $F_{(22,176)}=2.0, p=0.0072$, genotype: $F_{(2,16)}=1.99, p=0.1673$; time: $F_{(11,176)}=31.17$, $p<0.0001$; REMS: interaction: $F_{(22,176)}=2.85, p<0.0001$, genotype: $F_{(2,16)}=0.17, p=0.8497$; time: $F_{(11,176)}=$ 26.4, $p<0.0001$; REM/TS: interaction: $F_{(22,176)}=0.93, p=0.55$; genotype: $F_{(2,16)}=0.65, p=0.5377$; time: $F_{(5,75)}=$ $12.51, p<0.0001$; post hoc Tukey's multiple comparison tests ${ }^{*} p<0.05,{ }^{* *} p<0.01$, ${ }^{* *} p<0.001$ WT vs $S \mathrm{SN}^{\mathrm{Con}}$; $\mathrm{xp}<0.05, \mathrm{xxp}<0.01$ WT vs $\mathrm{SCN}^{\mathrm{Cr} 1} ;+p<0.05 \mathrm{SCN}^{\mathrm{Con}}$ vs $\left.\mathrm{SCN}^{\mathrm{Cr} y 1}\right)$. $\boldsymbol{E}-\boldsymbol{H}$, Two circadian hour profiles (mean \pm SEM) of wakefulness $(\boldsymbol{E})$, NREMS $(\boldsymbol{F})$, REMS $(\boldsymbol{G})$, and REMS/TS $(\boldsymbol{H})$ in WT ( $n=5$; blue), $\mathrm{SCN}^{\mathrm{Con}}$ mice $(n=6$; green), and $\mathrm{SCN}^{\mathrm{Cry} 1}$ ( $n=7$; magenta) under free-running constant conditions [note, not possible to do statistics including $\mathrm{SCN}^{\mathrm{Cry} 1}$ as these mice have a different endogenous free-running period and so a different timescale] $\left(2 \times\right.$ RMANOVA wake: interaction: $F_{(11,99)}=7.6, p<0.0001$, genotype: $F_{(1,9)}=5.073, p=0.0508$; time: $F_{(11,99)}=9.75, p<0.0001$; NREMS: interaction: $F_{(11,99)}=7.5, p<0.0001$, genotype: $F_{(1,9)}=2.5$, $p=0.1481$; time: $F_{(11,99)}=9.3, p<0.0001$; REMS: interaction: $F_{(11,99)}=4.6, p<0.0001$, genotype: $F_{(1,9)}=$ 5.26, $p=0.0474$; time: $F_{(11,99)}=7.5, p<0.0001$; REM/TS: interaction: $F_{(11,99)}=2.8, p=0.0033$, genotype: $F_{(1,9)}=5.84, p=0.0388$; time: $F_{(11,99)}=6.68, p<0.0001$; post hoc Tukey's multiple comparison test * ${ }^{* *}$, ${ }^{* * *} p<0.05,0.01,0.001$ WT vs $\left.\mathrm{SCN}^{\mathrm{Con}}\right)$. Gray shaded area represents darkness.

than in circadian night (as assessed by post hoc Sidak's and Tukey's multiple comparisons tests within genotype and between genotypes, respectively, where there was a significant interaction and/or genotype effect in a $2 \times$ RMANOVA; see legend to Fig. 6 for details). Thus, global loss of Cry proteins destabilizes sleepwake structure on LD and even more so under $\mathrm{DD}$, while restoring local expression of Cryl in the SCN region, resulting in a molecularly competent SCN, reverses the loss of sleep-wake architecture and consolidates vigilance states in $\mathrm{SCN}^{\mathrm{Cry} 1}$ mice.

These differences in sleep-wake consolidation between groups were emphasized further by the number of transitions between wake-NREMS and NREMS-wake (there were no significant differences in transitions between NREMS-REMS, REMS-NREMS or REMS-wake following a $1 \times$ ANOVA in either $\mathrm{LD}$ or DD). In both $\mathrm{LD}$ and $\mathrm{DD}, \mathrm{SCN}^{\mathrm{Con}}$ mice showed more transitions than did WT mice, and this was reversed in $\mathrm{SCN}^{\mathrm{Cry} 1}$ mice in both LD (Fig. 6E) and DD (Fig. 6F) compared with WT and $\mathrm{SCN}^{\mathrm{Cryl}}$ mice (as assessed by post hoc Tukey's multiple comparisons tests within genotype following a $1 \times$ ANOVA; see legend to Fig. 6 for details). This further confirms that rhythmic expression of Cryl targeted to the SCN of Cry1/Cry2-null mice stabilizes the sleep-wake over both the 24-h LD cycle and across CT, consistent with the view that the suprachiasmatic pacemaker drives the maintenance of wakefulness and the consolidation of sleep, as appropriate, across the LD cycle and across subjective day and night.

\section{Temporal control of sleep homeostasis in SCN ${ }^{\text {Cry1 }}$ mice}

To what extent can SCN-mediated consolidation of sleep timing and patterning affect homeostasis? EEG $\delta$ power $(1-4 \mathrm{~Hz})$ during NREMS is a commonly used index of sleep homeostasis, with higher levels indicating increased sleep need. Under LD, absolute and relative EEG $\delta$ power in NREMS (normalized to total power to correct for electrode placement) in WT mice declined spontaneously across the inactive light phase and increased during the dark phase (Fig. $7 A, B$ ), coincident with increased wake. This pattern was clearly under circadian regulation in DD (Fig. 7C,D). In contrast, $\mathrm{SCN}^{\mathrm{Con}}$ mice revealed only a lowamplitude pattern on LD, and no significant circadian pattern in DD (Fig. 7C,D). As reported previously (Wisor et al., 2002); however, Cry1/Cry2-null mice exhibited higher levels of absolute NREMS EEG $\delta$ power, in particular during the light/inactive phase when compared with WT mice (Fig. $7 A, C$ ). $\mathrm{SCN}^{\mathrm{Cry} 1}$ mice had a significant rhythm in relative $\delta$ power not only in LD but also in DD (Fig. $7 B$, $D$; as assessed by post hoc Dunnett's multiple comparisons tests against the time point before transition to lights off (LD)/activity onset (DD) following a $1 \times$ ANOVA; see legend to Fig. 7 for details). In LD, the peak amplitude of EEG $\delta$ power of $\mathrm{SCN}^{\mathrm{Cry} 1}$ mice was the same as in WT, and although in DD the peak amplitude was reduced compared with WT, it was nevertheless phased appropriately (Fig. 7D). This lower peak may reflect the slightly higher levels of NREMS in the early circadian active phase in DD compared with WT, a difference which was not evident in LD (Fig. 5B,F). Finally, all groups showed a similar latency to first NREMS episode $>100 \mathrm{~s}$ on the dark-to-light transition (latency to first NREMS episode $>100 \mathrm{~s}$ : WT $=$ $18.9 \pm 8.9 \mathrm{~min}, n=5, \mathrm{SCN}^{\mathrm{Con}}=18.0 \pm 6.8 \mathrm{~min}, n=7$; $\mathrm{SCN}^{\mathrm{Cry} 1}=$ $17.5 \pm 5.2 \mathrm{~min}, n=7 ; 1 \times$ ANOVA: $\left.F_{(2,16)}=0.01, p=0.99\right)$. Together, these data demonstrate that rescuing Cryl in the SCN region restores rhythmic expression of NREMS EEG $\delta$ power in both LD and DD, confirming the appropriate phasing and organization of sleep-wake across the 24-h/circadian cycle. SCN- 
A



B

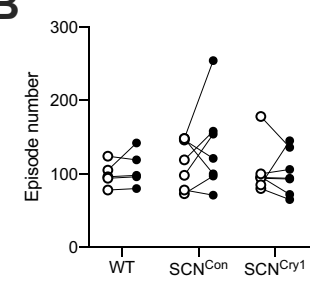

C

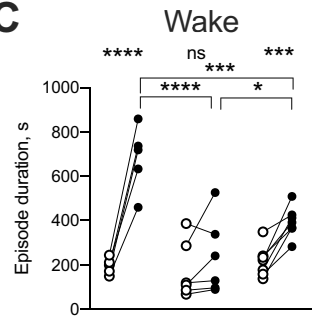

D



NREMS


NREMS
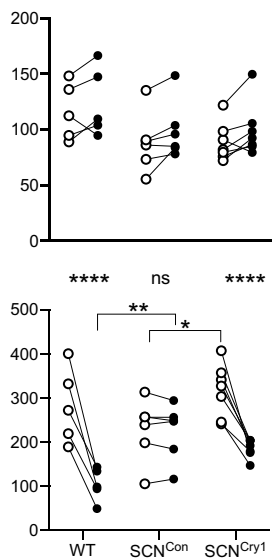

REMS
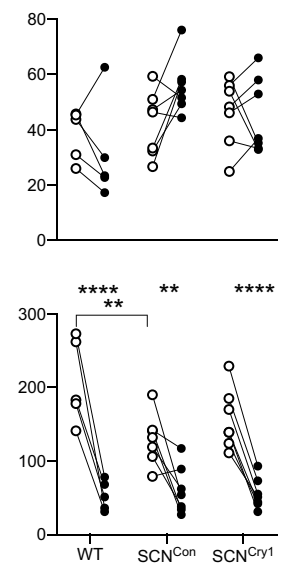

REMS
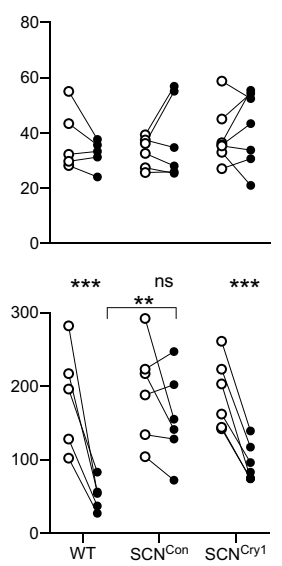

E

12L:12D

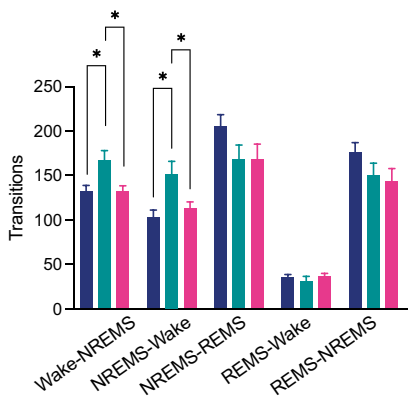

$\mathbf{F}$
DD

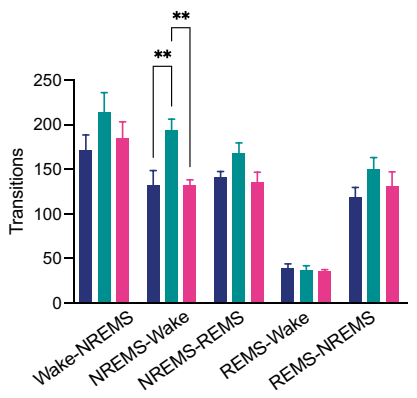

Figure 6. Local expression of Cry1 in the suprachiasmatic hypothalamus consolidates sleep/wake architecture. $A$, Duration (seconds) of bouts in wakefulness, NREM, and REM in $12 \mathrm{~h}$ light (open circles) and $12 \mathrm{~h}$ dark (closed circles) in WT ( $n=5$, blue), $\operatorname{SCN}^{\mathrm{Con}}\left(n=7\right.$, green), and $\mathrm{SCN}^{\mathrm{Cry} 1}\left(n=7\right.$, magenta) mice in entrained (LD) conditions. Whereas the WT and SCN $\mathrm{N}^{\mathrm{Cr} 11}$ mice have a significant increase in wake duration during the dark/active compared with the light/inactive phase, the $\mathrm{SCN}{ }^{\mathrm{Con}}$ animals have significantly fewer nocturnal bouts and so do not have a significant LD organization [episode duration: $2 \times$ RMANOVA wake: interaction: $F_{(2,16)}=8.5, p=0.003$; genotype: $F_{(2,16)}=1.97, p=0.1715$; time: $F_{(1,16)}=61.38, p<0.0001$; post hoc multiple com-

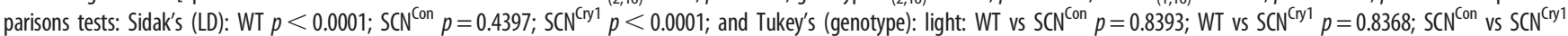
$p>0.9999$; dark: WT vs $S C N^{C o n} p=0.0044$; WT vs $S C N^{C r y 1} p=0.7266$; $\operatorname{SCN}^{\mathrm{Con}}$ vs $S_{C N}{ }^{\mathrm{Cry} 1} p=0.0161$; NREMS: interaction: $F_{(2,16)}=0.0665, p=0.9359 ;$ genotype: $F_{(2,16)}=4.393, p=0.0302$; time: $F_{(1,16)}=0.4075, p=0.5323$; post hoc multiple comparisons tests: Sidak's (LD): WT $p=0.9798$; SCN ${ }^{\mathrm{Con}} p=0.9991 ; \mathrm{SCN}^{\mathrm{Cry1}} p=0.8986$; and Tukey's (genotype): light: WT vs SCN ${ }^{\mathrm{Con}}$

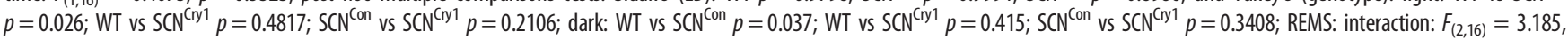
$p=0.0685$; genotype: $F_{(2,16)}=3.041, p=0.076$; time: $F_{(1,16)}=0.237, p=0.633$ ]. $B$, Number of bouts of wakefulness, NREMS and REMS in $12 \mathrm{~h}$ light (open circles) and $12 \mathrm{~h}$ dark (closed circles) in WT ( $n=5$, blue), $S_{C N}{ }^{\text {Con }}\left(n=7\right.$, green), and $\operatorname{SCN}^{\text {(ry) }}(n=7$, magenta) mice in LD. All three genotypes have a significant LD organization of NREMS and REMS episodes, although the $S \mathrm{SN}^{\mathrm{Con}}$ mice have fewer bouts of NREMS and REMS in the light period, i.e., the rest period [episode number: $2 \times$ RMANOVA wake: interaction: $F_{(2,16)}=0.635, p=0.5427 ;$ genotype: $F_{(2,16)}=$ 1.17, $p=0.335$; time: $F_{(1,16)}=0.93, p=0.341$; NREMS: interaction: $F_{(2,16)}=3.688, p=0.0482$; genotype: $F_{(2,16)}=1.779, p=0.2006$; time: $F_{(1,16)}=99.36, p<0.0001 ;$ post hoc multiple com-

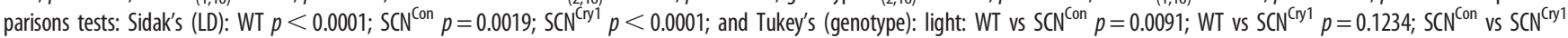
$p=0.4282$; dark: WT vs $S C C^{C o n} p=0.9661$; WT vs $S C N^{\mathrm{Cr} y 1} p=0.9778$; $\operatorname{SCN}^{\mathrm{Con}}$ vs $\mathrm{SCN}^{\mathrm{Cry} 1} p=0.9985$; REMS: interaction: $F_{(2,16)}=5.573, p=0.0146$; genotype: $F_{(2,16)}=2.259, p=0.1367$; time: $F_{(1,16)}=115.3, p<0.0001$; post hoc multiple comparisons tests: Sidak's (LD): WT $p<0.0001$; $\operatorname{SCN}^{\mathrm{Con}} p=0.0019 ; \mathrm{SCN}^{\mathrm{Cry1}} p<0.0001$; and Tukey's (genotype): light: WT vs SCN ${ }^{\mathrm{Con}} p=0.0023$; WT vs $S C N^{C r y 1} p=0.0543$; $S C N^{C o n}$ vs $S C N^{C r y 1} p=0.3507$; dark: WT vs $S C N^{C o n} p=0.9347$; WT vs $S C N^{C r y 1} p=0.9904$; $S C N^{C o n}$ vs $\left.S C N^{C r y 1} p=0.969\right]$. C, Duration (seconds) of bouts in wakefulness, 
mediated circadian organization and consolidation were therefore accompanied by appropriate dynamic signaling of sleep need.

Having shown that the suprachiasmatic clock can direct the circadian patterning and stabilization of sleep-wake cycles, we next tested whether restoring a molecularly competent clock in the SCN region has an effect on the homeostatic regulation of sleep by measuring sleep and the EEG responses following 6-h SD that started from lights onset (ZT0-ZT6). SD was equally effective across the three groups, with no significant differences in the small amount of NREMS during SD (WT $9.8 \pm 3.5 \mathrm{~min}, \mathrm{SCN}^{\mathrm{Con}} 13.6 \pm$ $2.7 \mathrm{~min}, \mathrm{SCN}^{\mathrm{Cry1}} 7.4 \pm 2.5 \mathrm{~min}, n=5,7,7$, respectively; $1 \times$ ANOVA: $\left.F_{(2,16)}=1.61, p=0.2311\right)$ or the latency to sleep after SD (time to first NREMS bout $>100$ s duration, WT $12.9 \pm 6.8 \mathrm{~min} ; \mathrm{SCN}^{\mathrm{Con}} 10.4 \pm 3.4 \mathrm{~min} ; \mathrm{SCN}^{\mathrm{Cry} 1} 10.3 \pm 3.7 \mathrm{~min}$; $1 \times$ ANOVA: $\left.F_{(2,16)}=0.18, p=0.8354\right)$. In the $2 \mathrm{~h}$ immediately after

NREM, and REM in subjective day (open circles) and subjective night (closed circles) in WT ( $n=5$, blue), $\mathrm{SCN}^{\mathrm{Con}}\left(n=6\right.$, green), and $\mathrm{SCN}^{\mathrm{Cry} 1}$ ( $n=7$, magenta) mice in free-running conditions (DD). Both the WT and $\mathrm{SCN}^{\mathrm{Cry} 1}$ mice have a significant circadian organization to wake duration but the $\mathrm{SCN}^{\mathrm{Con}}$ animals do not, because of significantly fewer wake episodes during the circadian night/active phase when compared with the WT and $\mathrm{SCN}^{\mathrm{Cr} 11}$ mice [episode duration: $2 \times$ RMANOVA wake: interaction: $F_{(2,16)}=26.33, p<0.0001$; genotype: $F_{(2,16)}=$ 7.14, $p=0.0066$; time: $F_{(1,16)}=104.4, p<0.0001$; post hoc multiple comparisons tests: Sidak's (LD): WT $p<0.0001 ;$ SCN ${ }^{\text {Con }} p=0.3901 ; S C^{C r y 1} p=0.0008$; and Tukey's (genotype): light: WT vs SCN ${ }^{\text {Con }} p=0.9564$; WT vs $S C N^{\text {Cry1 }} p=0.9421$; $\mathrm{SCN}^{\text {Con }}$ vs SCN ${ }^{\text {Cry }} p=7902$; dark: WT vs $\mathrm{SCN}^{\mathrm{Con}} p<0.0001$; WT vs $S \mathrm{CN}^{\mathrm{Cry} 1} p=0.0004$; $\mathrm{SCN}^{\mathrm{Con}}$ vs $\mathrm{SCN}^{\mathrm{Cry} 1} p=0.0472$; NREMS: interaction: $F_{(2,16)}=0.072, p=0.9306$; genotype: $F_{(2,16)}=2.17, p=0.1485$; time: $F_{(1,16)}=$ 11.19, $p=0.044$; REMS: interaction: $F_{(2,16)}=1.543, p=0.2458$; genotype: $F_{(2,16)}=0.5545$, $p=0.5857$; time: $\left.F_{(1,16)}=0.089, p=0.792\right]$. $D$, Number of bouts of wakefulness, NREMS and REMS in subjective day (open circles) and subjective night (closed circles) in WT ( $n=5$, blue), $\mathrm{SCN}^{\mathrm{Con}}$ ( $n=6$, green), and $\mathrm{SCN}^{\mathrm{Cry} 1}$ ( $n=7$, magenta) mice in DD. In the absence of light, the $\mathrm{SCN}^{\mathrm{Con}}$ mice no longer show a circadian organization in the number of NREMS and REMS episodes, with significantly more episodes in the dark/active period compared with the WT mice [episode number: $2 \times$ RMANOVA wake: interaction: $F_{(2,16)}=0.8968, p=0.4266$; genotype: $F_{(2,16)}=4.52, p=0.0291$; time: $F_{(1,16)}=4.714, p=0.0464$; post hoc multiple comparisons tests: Sidak's (LD): WT $p=0.29569 ; \mathrm{SCN}^{\mathrm{Con}} p=0.2809 ; \mathrm{SCN}^{\mathrm{Cry} 1} p=0.09,948$; and Tukey's (genotype): light: WT vs SCN ${ }^{\mathrm{Con}} p=0.0272$; WT vs SCN ${ }^{\mathrm{Cry} 1} p=0.95879 ; \mathrm{SCN}^{\mathrm{Con}}$

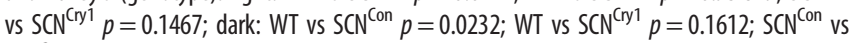
$\mathrm{SCN}^{\mathrm{Cry} 1} p=0.54$; NREMS: interaction: $F_{(2,16)}=19.42, p<0.0001$; genotype: $F_{(2,16)}=1.756$, $p=0.2065$; time: $F_{(1,16)}=80.45, p<0.0001$; post hoc multiple comparisons tests: Sidak's (LD): WT $p<0.0001 ; \mathrm{SCN}^{\mathrm{Con}} p=0.9967$; SCN ${ }^{\mathrm{ryy} 1} p<0.0001$; Tukey's (genotype): light: WT vs $S_{C N}{ }^{\text {Con }} p=0.294$; WT vs $S_{C N}{ }^{\text {Cry } 1} p=0.5757 ;$ SCN $^{\text {Con }}$ vs SCN ${ }^{\text {Cry } 1} p=0.0279$; dark: WT vs $\mathrm{SCN}^{\mathrm{Con}} p=0.0056$; WT vs SCN ${ }^{\mathrm{Cr} 1} p=0.058 ; \mathrm{SCN}^{\mathrm{Con}}$ vs SCN ${ }^{\mathrm{Cry} 1} p=0.4883$; REMS: interaction: $F_{(2,16)}=4.184, p=0.036$; genotype: $F_{(2,16)}=2.032, p=0.1656$; time: $F_{(1,16)}=42.26$, $p<0.0001$; post hoc multiple comparisons tests: Sidak's (LD): WT $p=0.0006$; SCN $^{\text {Con }}$ $p=0.4367$; $\mathrm{SCN}^{\mathrm{Cry} 1} p=0.0005$; Tukey's (genotype): light: WT vs $\mathrm{SCN}^{\mathrm{Con}} p=0.9696$; WT vs $\mathrm{SCN}^{\mathrm{Cry} 1} p=0.7682 ; \mathrm{SCN}^{\mathrm{Con}}$ vs SCN ${ }^{\mathrm{Cry} 1} p=0.8841$; dark: WT vs $\mathrm{SCN}^{\mathrm{Con}} p=0.0103$; WT vs

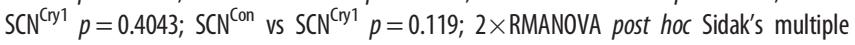
comparisons test (LD difference within genotype) ${ }^{* * * *} p<0.0001,{ }^{* * *} p<0.001$, ${ }^{* *} p<0.01$; post hoc Tukey's multiple comparisons test (genotype difference) ${ }^{* * * *} p<0.0001{ }^{* * *} p<0.001,{ }^{* *} p<0.01,{ }^{*} p<0.05$ ]. $E$, Mean (+ SEM) number of transitions between sleep-wake states in $L D$ reveals an increase in the numbers of transitions between wake-NREMS-wake in the $\mathrm{SCN}^{\mathrm{Con}}$ mice suggesting a lack of consolidated sleep/wake $\left(1 \times\right.$ ANOVA: LD: wake-NREMS: $F_{(2,16)}=5.8, p=0.012$; Tukey's multiple comparisons test WT vs $\mathrm{SCN}^{\text {Con }} p=0.033$; WT vs $\mathrm{SCN}^{\text {Cry1 }} p=0.9996 ; \mathrm{SCN}^{\text {Con }}$ vs SCN ${ }^{\text {Cry } 1}$ $p=0.021$ : NREMS-wake: $F_{(2,16)}=5.5, p=0.0148$; Tukey's multiple comparisons test WT vs $S_{C N}{ }^{\text {Con }} p=0.0203$; WT vs $S C N^{\text {Cryy }} p=0.788 ;$ SCN $^{\text {Con }}$ vs SCN $\left.{ }^{\text {Cry1 }} p=0.0481\right)$. F, Mean (+ SEM) number of transitions between sleep-wake states in DD shows a significant increase in NREMS-wake transitions in the $\mathrm{SCN}^{\mathrm{Con}}$ mice compared with both the WT and $\mathrm{SCN}^{\mathrm{Cry} 1}$ mice. Therefore, restoration of rhythmicity to the region of the $\mathrm{SCN}$ in SCN ${ }^{\text {ry } 1}$ mice restores sleep/wake consolidation $\left(1 \times\right.$ ANOVA: DD: wake-NREMS: $F_{(2,15)}=1.1$, $p=0.3$; NREMS-wake: $F_{(2,15)}=10.8 p=0.0012$; Tukey's multiple comparisons test WT vs $\mathrm{SCN}^{\text {Con }} p=0.0044 ;$ WT vs $\mathrm{SCN}^{\text {Cry } 1} p=0.9995 ; \operatorname{SCN}^{\text {Con }}$ vs $\operatorname{SCN}^{\text {Cry1 }} p=0.0021$; ${ }^{*} p<0.05,{ }^{* *} p<0.01$.
$\mathrm{SD}$, all groups showed a significant increase in $\delta$ power when NREMS occurred in that interval reflecting greater homeostatic sleep pressure (Fig. 8A; no significant interaction or genotype effect following $2 \times$ RMANOVA showing that all genotypes responded to the 6-h SD in the same way; see legend to Fig. 8 for statistical analyses). Genotype did not, therefore, affect the neurophysiological capacity to sense and respond to SD during subsequent NREMS when it did occur (Wisor et al., 2002). Furthermore, recovery from $\mathrm{SD}$, compared with baseline, was not different between genotypes, insofar as accumulated sleep loss increased during SD, but then decreased at the same rate in all three groups over the subsequent $18 \mathrm{~h}$ (Fig. 8B). In addition, over the $6 \mathrm{~h}$ immediately following SD (ZT6-ZT12), the genotype of the mice did not significantly affect the change in NREMS bout duration compared with baseline, although the individual $\mathrm{WT}$ and $\mathrm{SCN}^{\mathrm{Cry} 1}$ mice all showed an increase following 6-h SD, the response was more variable in the $\mathrm{SCN}^{\mathrm{Con}}$ animals (Fig. $8 \mathrm{C}$; no significant interaction or genotype effect following $2 \times$ RMANOVA; see legend to Fig. 8 for statistical analyses; Fig. $8 C$ ). By whatever mechanism, all three groups recovered lost sleep, i.e., exhibited effective homeostasis.

Notwithstanding overall comparability between genotypes, there were also informative differences. The time course for the decline in EEG $\delta$ power in NREMS after SD was different. It declined progressively across the light phase in WT mice, but more slowly in $\mathrm{SCN}^{\mathrm{Con}}$ mice and, unlike in WT mice, did not reach baseline levels during the light phase (Fig. 8D-F). Expression of Cryl in the SCN region corrected these deficits (Fig. 8C,D,G). Thus, although there were no significant differences in the overall recovery of sleep loss after SD (Fig. $8 B$ ), there were significant differences in its time course during the light phase (ZT6-ZT12; Fig. $8 H-J)$. Whereas WT and SCN ${ }^{\mathrm{Cry} 1}$ mice showed a sustained absence of wake (Fig. $8 H$ ) and elevation of both NREMS and REMS in the light phase (Fig. 8I,J), the $\mathrm{SCN}^{\mathrm{Con}}$ mice exhibited significantly less $(\sim 70 \mathrm{~min})$ NREMS and more ( $\sim 60 \mathrm{~min}$ ) wakefulness between ZT6-ZT12 following 6-h SD $\left(1 \times\right.$ ANOVA: wake: $F_{(2,16)}=6.72, p=0.0076$, Tukey's post hoc multiple comparisons tests: WT vs $\mathrm{SCN}^{\mathrm{Con}} p=0.017$, WT vs $\mathrm{SCN}^{\mathrm{Cry} 1} p=0.9527, \mathrm{SCN}^{\mathrm{Con}}$ vs $\mathrm{SCN}^{\mathrm{Cry1}} p=0.017$; NREMS: $F_{(2,16)}=5.76, p=0.013$, Tukey's post hoc multiple comparisons tests: WT vs $\mathrm{SCN}^{\mathrm{Con}} p=0.034$, WT vs $\mathrm{SCN}^{\mathrm{Cry} 1} p=0.9995$, $\mathrm{SCN}^{\mathrm{Con}}$ vs $\left.\mathrm{SCN}^{\mathrm{Cry} 1} p=0.022\right)$. This suggests that $\mathrm{SCN}^{\mathrm{Con}}$ mice had a decreased sleep pressure and/or an inability to maintain consolidated NREMS at this phase of the LD cycle. Given that analysis of NREMS EEG $\delta$ power indicated that decreased sleep pressure was not the case in $\mathrm{SCN}^{\mathrm{Con}}$ mice (Fig. $8 A, D, F$ ), consolidation of NREMS bout duration may have been limiting, as all WT and SCN ${ }^{\mathrm{Cry} 1}$ mice increased the NREMS bout duration following 6-h SD (Fig. $8 \mathrm{C}$ ), overall the $\mathrm{SCN}^{\mathrm{Con}}$ mice did not show a significant difference in this parameter \pm 6 -h SD (two-tailed paired $t$ test $t_{(6)}=1.15, p=0.2925$; Fig. $8 C$ ). It may be that poor consolidation and/or less time in NREMS at this phase (ZT6ZT12) altered the time course to recovering sleep loss, and may reflect an interaction between the circadian and homeostatic processes regulating sleep-wake at the end of the light phase (Fig. $8 D, F, I)$. Loss of Cry proteins did not, therefore, globally affect neurophysiological mechanisms of homeostatic sleep recovery, but in $\mathrm{SCN}^{\mathrm{Con}}$ mice with an ineffective SCN clock, the dynamics of recovery were altered and the expression of Cryl in the SCN region corrected this (Fig. $8 \mathrm{C}-J$ ). Cry proteins and a competent SCN clock are not, therefore, necessary components of the fundamental sleep homeostatic mechanism, but they do regulate its time course. 


\section{Rescue of sleep-dependent memory in the novel object test in $\mathrm{SCN}^{\mathrm{Cry1}}$ mice} To determine whether SCN-mediated circadian control over phasing and consolidation of sleep/wake cycles has consequences for brain function, we assessed cognitive performance in the NOR task, a recognized sleep-dependent behavior (Palchykova et al., 2006). There were no statistically significant differences between groups in time spent exploring the objects during training (Fig. 9A,B; $2 \times$ RMANOVA: interaction: $F_{(2,16)}=0.4099, p=0.6705$; object: $F_{(1,16)}=0.1558, \quad p=0.6982$; group: $\left.F_{(2,16)}=0.8779, p=0.4348\right)$. WT mice demonstrated robust memory for the familiar object by spending significantly more time investigating the novel object when tested (positive DI). In contrast, $\mathrm{SCN}^{\mathrm{Con}}$ mice failed to discriminate between the novel and familiar objects, with an overall null preference between the two objects (Fig. 9C). Thus, the global absence of Cry proteins compromised performance in a test of memory known to be sleep-dependent. The initiation of circadian competence in $\mathrm{SCN}^{\mathrm{Cry} 1}$ mice, and thereby organization of sleep/wake cycles, resulted in all seven $\mathrm{SCN}^{\mathrm{Cry} 1}$ mice showing a preference for the novel object $\left(1 \times\right.$ ANOVA $F_{(2,16)}=$ $11.58, p=0.0008$; post hoc Tukey's multiple comparison test: WT vs $\mathrm{SCN}^{\text {Con }}$ $p=0.0015$, WT vs $\mathrm{SCN}^{\mathrm{Cry} 1} p=0.7493$, $\mathrm{SCN}^{\mathrm{Con}}$ vs $\left.\mathrm{SCN}^{\mathrm{Cry} 1} p=0.0035\right)$. These results demonstrate that global loss of Cryl proteins compromises NOR performance, and that a molecularly competent suprachiasmatic pacemaker cannot only establish the necessary organization and consolidation of sleep/wake, but also sustain sleep-dependent memory.

\section{Discussion}

To address the specific role of the SCN in sleep regulation we used genetically clockless Cry1/Cry2-null mice that in the absence of LD have no circadian patterning to sleep-wake cycles, poorly consolidated sleep and wake, compromised dynamics of homeostatic recovery sleep, and impaired sleep-dependent memory (van der Horst et al., 1999; Wisor et al., 2002, 2008; Maywood et al., 2018). We initiated de novo circadian rhythmicity locally to the SCN and closely adjacent hypothalamus by virally mediated expression of Cry1 (Edwards et al., 2016; Maywood et al., 2018). Importantly, the SCN was the only tissue successfully targeted in all mice, but for caution we refer to the SCN region. The rest of the brain, and the periphery remained circadian incompetent. In agreement with previous studies we established circadian locomotor activity rhythms in $\mathrm{SCN}^{\mathrm{Cry} 1}$ mice (Maywood et al., 2018) comparable to the effect of WT SCN grafts in Cry1/Cry2-null mice (Sujino et al., 2003). This allowed us to test the contribution of the suprachiasmatic magenta.
B
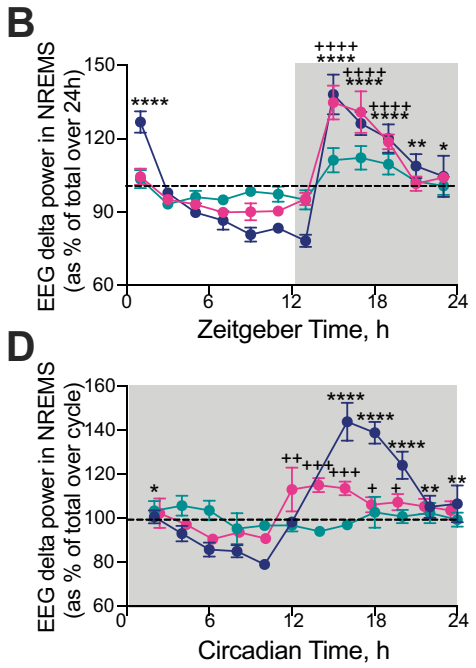

Figure 7. Characterization of the effect of local Cry1 expression in the suprachiasmatic hypothalamus on $\delta$ power (1-4 $\mathrm{Hz}$ ) in NREMS in entrained and free-running conditions. $\boldsymbol{A}, 2$-h profiles (mean \pm SEM) of NREMS EEG raw $\delta$ power in WT,

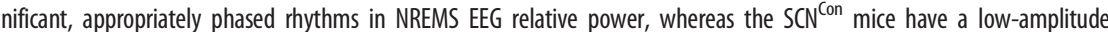
(WT: $1 \times$ ANOVA: $F_{(11,47)}=15.8, p<0.0001$; post hoc Dunnett's multiple comparisons test vs ZT10: $p<0.0001$ ZT2, versus ZT10: $p=0.1387 \mathrm{ZT2}, p=0.9811 \mathrm{ZT} 4, p=0.9993 \mathrm{ZT} 6, p>0.9999 \mathrm{ZT} 8, p>0.9999 \mathrm{ZT} 12, p=0.9815 \mathrm{ZT} 14$ $p<0.0001$ ZT16, $p<0.0001$ ZT18, $p<0.0001$ ZT20, $p=0.3489$ ZT22, $p=0.1845$ ZT24). C, 2-h profiles (mean \pm SEM) 0

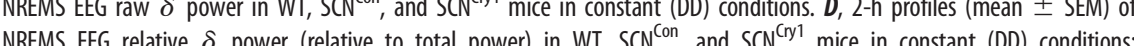
$1 \times$ ANOVA shows both WT and $\mathrm{SCN}^{\mathrm{Cry} 1}$ mice have significant circadian rhythms, but there was no significant rhythm in control-treated SCN ${ }^{\text {Con }}$ mice (WT: $F_{(11,40)}=20.1, p<0.0001$; post hoc Dunnett's multiple comparisons test versus CT10: $p=0.019$ CT2, $p=0.2632$ CT4, $p=0.9361$ CT6, $p=0.971$ (T8, $p<0.0001$ CT14-CT20, $p=0.0028$ CT22, $p=0.0032$ (T24); $F_{(11,58)}=0.78, p=0.66 ; S_{C N}{ }^{\text {ry1 }}: F_{(11,68)}=4.17, p<0.0001$, post hoc Dunnett's multiple comparisons test vs CT10: $++++p<0.0001$. Gray shaded area represents darkness; WT $(n=5)$, blue; $\operatorname{SCN}^{\mathrm{Con}}(n=7)$, green; $\operatorname{SCN}^{\mathrm{Cry} 1}(n=7)$,

molecular clockwork to the temporal regulation of sleep. Genetic rescue established sleep-wake cycles that were appropriately phased and consolidated, and accompanied by improved performance in a test of sleep-dependent memory. On some measures $\mathrm{SCN}^{\mathrm{Cryl}}$ mice did not reach those of WTs, which may reflect the incomplete targeting of the paired SCN. Nevertheless, the data suggest that the circadian system promotes wake in subjective night and facilitates sleep, by promoting its intensity and consolidation, in subjective daytime. Although the neural circuits and neurophysiological processes underlying sleep homeostasis are not compromised by global Cry 1,2 deficiency, our data do illustrate an interaction between the homeostatic and circadian mechanisms during the recovery from SD. We conclude that the SCN has continuous influence on sleep-wake organization across the circadian cycle and, directly or indirectly, modulates sleep consolidation and homeostatic regulation. Further dissection of the relevant neural pathways would require Cre-dependent targeting of specific subpopulations, such as VIP and VIP- or dopamine-receptor-expressing cells (Patton et al., 2020; Hamnett et al., 2021; Maywood et al., 2021) within the SCN and/or its targets. Moreover, analysis of female mice, in which the SCN direct estrous variability in arousal state, may provide additional insight. 
A

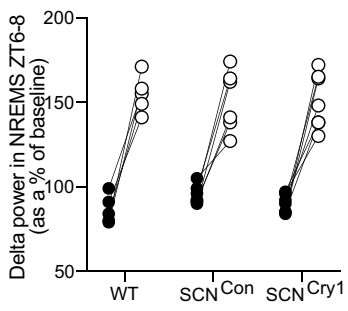

C

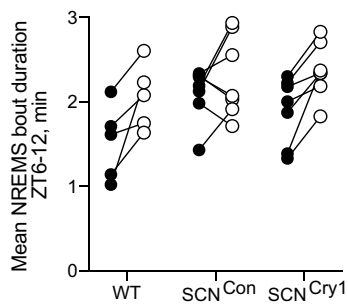

E

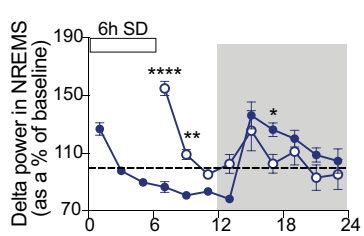

H

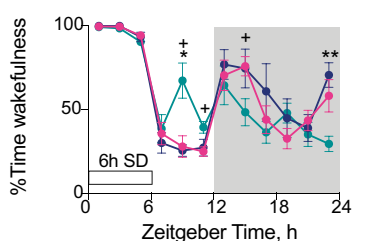

B

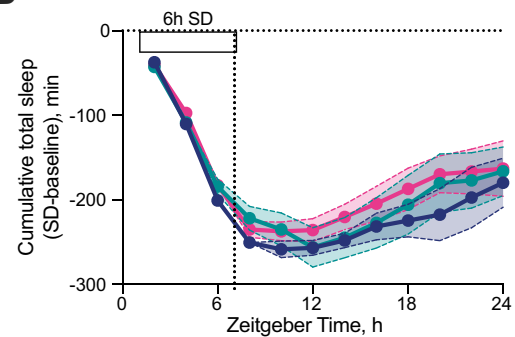

D



$\mathbf{F}$

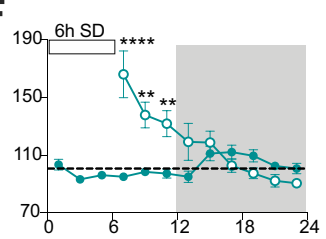

I

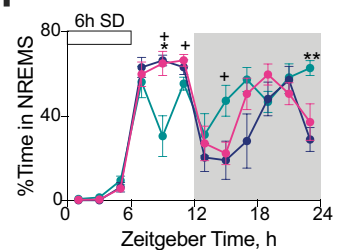

G

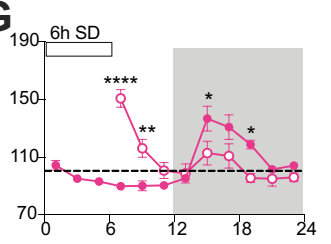

J

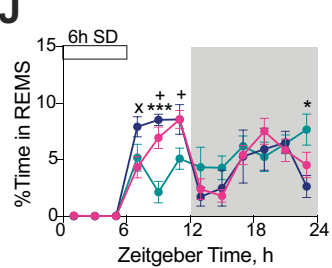

Figure 8. Effect of rescue of rhythmic Cry1 expression in the suprachiasmatic hypothalamus on the homeostatic response to 6h SD. $A$, Individual changes in NREMS $\delta$ power (1-4 Hz) in the first $2 \mathrm{~h}$ of recovery sleep (ZT6-ZT8; open circles) compared with baseline sleep (ZT6-ZT8; closed circles) in WT, $\mathrm{SCN}^{\mathrm{Con}}$, and SCN ${ }^{\mathrm{Cry} 1}$ mice shows a significant effect of the treatment (6-h SD) with all three genotypes showing the SD was effective in initiating a significant homeostatic response, but no genotype or genotype $\times$ treatment effects ( $2 \times$ RMANOVA: interaction: $F_{(2,16)}=0.19, p=0.83$; genotype: $F_{(2,16)}=1.19, p=0.33$; treatment $\left( \pm 6-\mathrm{h}\right.$ SD): $F_{(1,16)}=83.91$, $p<0.0001)$. $\boldsymbol{B}$, No significant differences between genotypes in the cumulative decrease in TS time (Mean \pm SEM; NREMS + REMS) over the $6 \mathrm{~h}$ of SD with subsequent partial recovery of sleep during the $18 \mathrm{~h}$ post-SD in WT ( $n=5$, blue), SCN ${ }^{\mathrm{Con}}(n=7$, green), and $\mathrm{SCN}^{\mathrm{Cr} 1}\left(n=7\right.$, magenta) mice $\left(2 \times\right.$ RMANOVA: interaction: $F_{(22,176)}=0.39, p=0.99$; genotype: $F_{(2,16)}=0.43, p=0.65$; time: $F_{(2,24)}=$ $58.5, p<0.0001)$. $C$, Individual changes in NREMS bout duration (minutes) between ZT6 and ZT12 on baseline day (closed circles) and after 6-h SD (open circles); although there was a significant effect of the treatment (6-h SD) in increasing the duration of the NREMS bout duration there was no genotype or genotype $\times$ treatment effect $\left(2 \times\right.$ RMANOVA: interaction: $F_{(2,16)}=1.3, p=0.29$; genotype: $F_{(2,16)}=2.25, p=0.14$; treatment $\left.( \pm 6-\mathrm{h} \mathrm{SD}): F_{(1,16)}=20.15, p=0.0004\right)$. $\boldsymbol{D}$, Comparison of the time course of EEG $\delta$ power (Mean \pm SEM) in NREMS following $6 \mathrm{~h}$ of SD between WT (blue), SCN ${ }^{\mathrm{Con}}$ (green), and SCN ${ }^{\mathrm{Cry} 1}$ (magenta) mice. The decline in EEG $\delta$ power in NREMS was significantly slower in $\mathrm{SCN}^{\mathrm{Con}}$ mice when compared with WT and SCN ${ }^{\mathrm{Cr} 1}$ animals ( $2 \times$ RMANOVA: interaction: $F_{(16,123)}=2.33, p=0.0049$, genotype: $F_{(2,16)}=0.91, p=0.4235$; time: $F_{(4,55)}=27.22, p<0.0001$; post hoc Tukey's multiple comparison test ${ }^{*} p<0.05,{ }^{* *} p<0.01$ WT vs $\mathrm{SCN}^{\mathrm{Con}} ;++p<0.01 \mathrm{SCN}^{\mathrm{Con}}$ vs $\mathrm{SCN}^{\mathrm{Cry} 1}$ ). E-G, Time course (mean \pm SEM) of EEG $\delta$ power relative to baseline during NREMS on baseline day (closed circles) and following 6-h SD (open circles; data replotted from $D$ )

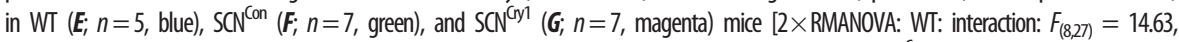

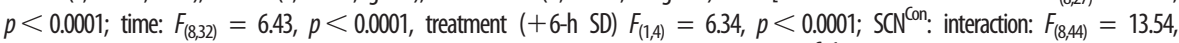
$p<0.0001$; time: $F_{(8,48)}=7.54, p<0.0001$, treatment $(+6-\mathrm{h} \mathrm{SD}) F_{(1,6)}=6.0, p=0.0498$; SCN $^{\mathrm{Cry} 1}$ : interaction: $F_{(8,45)}=17.84, p<0.0001$; time: $F_{(8,48)}=12.14, p<0.0001$, treatment $\left(+6-\mathrm{h}\right.$ SD) $F_{(1,6)}=60.28, p=0.613$; post hoc Sidak's multiple comparison test baseline vs $+6-\mathrm{h}$ SD $\left.{ }^{*} p<0.05,{ }^{* *} p<0.01,{ }^{* *} p<0.001,{ }^{* * *} p<0.0001\right]$. $\boldsymbol{H}-\boldsymbol{J}$, Percentage time (mean \pm SEM) spent in wakefulness, NREMS and REMS during $6 \mathrm{~h}$ of SD and $18 \mathrm{~h}$ of recovery in WT ( $n=5$, blue), $\mathrm{SCN}^{\mathrm{Con}}\left(n=7\right.$, green), and SCN ${ }^{\mathrm{Cr} 1}\left(n=7\right.$, magenta) mice. The SCN ${ }^{\mathrm{Con}}$ mice show significant increases in wake with concomitant decreases in NREMS and REMS during the recovery from 6-h SD, in particular during the light phase (ZT6-ZI12), and at the end of the dark phase ( $2 \times$ RMANOVA: wake: interaction: $F_{(22,176)}=3.05, p<0.0001$, genotype: $F_{(2,16)}=$ $0.76, p=0.49$; time: $F_{(6,89)}=40.96, p<0.0001$; NREMS: interaction: $F_{(22,176)}=3.43, p<0.0001$, genotype: $F_{(2,16)}=1.54, p=0.2436$; time: $F_{(6,97)}=46.45, p<0.0001$; REMS: interaction: $F_{(22,176)}=2.97, p<0.0001$, genotype: $F_{(2,16)}=0.11, p=0.8982 ;$ time: $F_{(6,93)}=24.01$, $p<0.0001$; post hoc Tukey's multiple comparison test ${ }^{*} p<0.05$, ${ }^{* *} p<0.01$, ${ }^{* * *} p<0.001$ WT vs SCN ${ }^{\text {Con }}$; $x p<0.05$ WT vs SCN ${ }^{\mathrm{Cry} 1}$; $+p<0.05 \mathrm{SCN}^{\mathrm{Con}}$ vs SCN $\left.{ }^{\mathrm{Cry} 7}\right)$.

Overall sleep-wake distributions across the entrained and free-running cycles showed modest differences between groups, with Cry1,Cry2null mice $\left(\mathrm{SCN}^{\mathrm{Con}}\right.$ and $\mathrm{SCN}^{\mathrm{Cry1}}$ ) showing an overall increase in the amount of NREMS in LD (Wisor et al., 2002). Studies using other global circadian mutations/deletions or ablation of the SCN in otherwise intact animals (Naylor et al., 2000; Easton et al., 2004; Laposky et al., 2005; Mistlberger, 2005) have demonstrated either no differences or an increase/decrease in NREMS, making it difficult to interpret whether any phenotypes are because of an extra-SCN circadian effect, or a more global effect on the dynamics of the complex neural circuitry underlining the control of sleep-wake states (Saper et al., 2010). Nevertheless, the SCN ${ }^{\mathrm{Con}}$, but not the SCN ${ }^{\text {Cryl }}$ mice, did show a significant decrease in the amount of NREMS in the second half of the light/ rest phase, suggesting that restoring rhythmicity to the SCN enables promotion of sleep/inhibition of wake at a time when the homeostatic pressure to sleep has declined. Furthermore, whereas the rescued animals showed a WT-like consolidation of wake episodes in the dark/active phase in both $\mathrm{LD}$ and $\mathrm{DD}$, the $\mathrm{SCN}{ }^{\mathrm{Con}}$ mice did not, and this was reflected in their reduced amplitude or absence of rhythmic time course for NREMS $\delta$ power in LD and $\mathrm{DD}$, respectively. Together, these results show the mouse SCN clock has opposing influences on sleep-wake organization across the cycle: promoting wakefulness in the dark/subjective day and sleep in light/subjective night.

The dynamics of sleep homeostasis were examined following SD, to assess whether there is an interaction between circadian and homeostatic regulatory processes. Evidence suggests influences of sleep homeostasis on the functioning of the circadian clock (Deboer et al., 2003, 2007; Schmidt et al., 2009), and that these processes can act independently (Tobler et al., 1983; Shiromani et al., 2004). Conversely, global circadian clock mutants can also show altered changes in NREMS EEG $\delta$ power in response to $\mathrm{SD}$, consistent with a role for clock genes in sleep homeostasis (Naylor et al., 2000; Wisor et al., 2002; Laposky et al., 2005; Dijk and Archer, 2010; Curie et al., 2013). As discussed above, however, this does not 
A

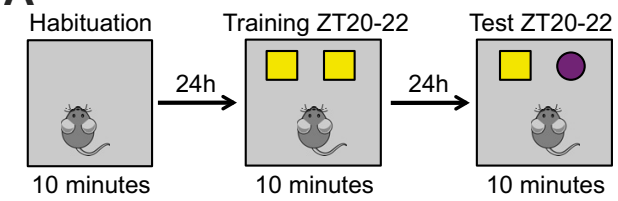

B

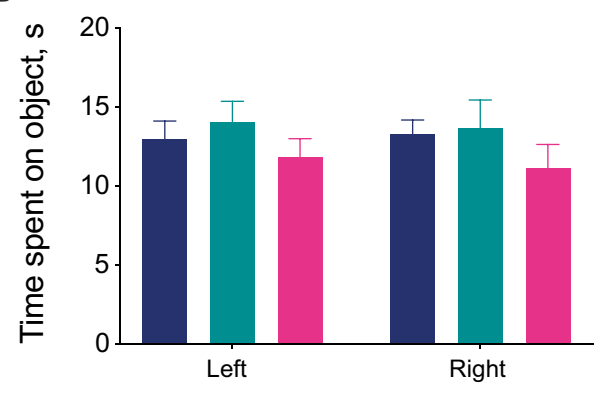

C

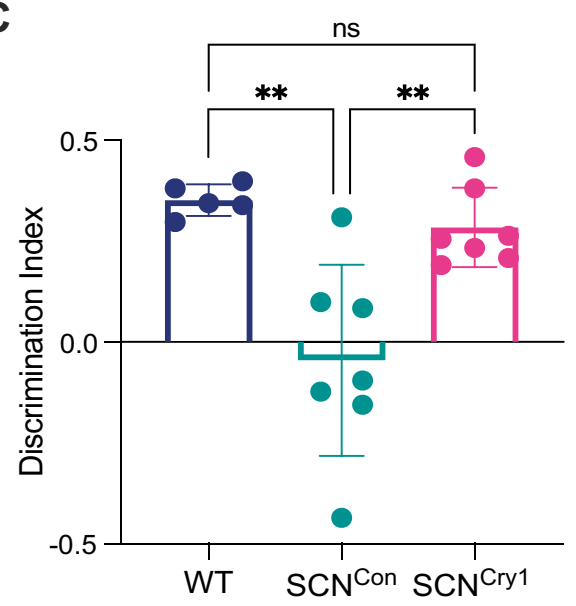

Figure 9. Cry1 expression in the suprachiasmatic hypothalamus rescues performance in the novel object test. $\boldsymbol{A}$, Protocol for the sleep-dependent memory test where mice are habituated to the test arena; $24 \mathrm{~h}$ later, mice investigate two identical objects, then $24 \mathrm{~h}$ later, one object is replaced with a novel object. Training and testing were done during the dark/ active phase between ZT20 and ZT22. $\boldsymbol{B}$, The amount of time (mean \pm SEM) the mice spent on the objects during the training phase was not significantly different between groups (see Results). C, The DI (mean \pm SEM and individual points) revealed the $\mathrm{SCN}^{\text {Con }}$ mice had an overall null preference for the novel object, whereas both the WT and $\mathrm{SCN}^{\mathrm{Cr} 1}$ groups of mice showed a significant preference for the novel object (see Results; $1 \times$ ANOVA with post hoc Tukey's multiple comparison test: WT vs $\mathrm{SCN}^{\mathrm{Con} * *} p<0.01$, WT vs $\mathrm{SCN}^{\text {Cry1 }}$ n.s., $\mathrm{SCN}^{\mathrm{Con}}$ vs $\left.\mathrm{SCN}^{\mathrm{Cry} 1 * *} p<0.01\right)$; WT $(n=5)$, blue; $\mathrm{SCN}^{\mathrm{Con}}(n=7)$, green; $\mathrm{SCN}^{\mathrm{Cr} y 1}(n=7)$, magenta.

necessarily indicate a role for the SCN in regulating homeostasis. Indeed, Cry1,Cry2-null mice, and mice lacking the Cry2 gene $\left(\mathrm{SCN}^{\mathrm{Cryl}}\right)$ have an intact sleep homeostatic response (Wisor et al., 2002, 2008), suggesting that the SCN is not necessary for the expression of the initial neurophysiological response to SD. Nevertheless, $\mathrm{SCN}^{\mathrm{Con}}$ mice had an altered time course in their recovery from SD, most notably between ZT6 and ZT12 when the SCN is exerting a sleep-promoting influence. This suggests a role, direct or indirect, for the circadian timing system in modulating the recovery from SD, and implies an interaction between the homeostatic and circadian processes, to ensure prolonged and consolidated sleep at a phase when sleep pressure is low. Studies in humans have similarly postulated a role for the circadian system in influencing sleep homeostasis (Lazar et al., 2015), although it remains to be established whether the central circadian clock in the SCN, and/or clocks in other brain areas underlie these mechanisms.
How might the SCN exert its effects? The sleep-wake regulatory circuit has been described as a "flip-flop" switch, whereby sleep-wake transitions are regulated by a reciprocal inhibition between sleep-promoting and wake-promoting nodes of the hypothalamus and brainstem (Saper et al., 2010). Multiple direct and indirect pathways from the SCN to both sleep-promoting or wake-promoting nodes could therefore influence state-switching over the circadian cycle, which would be expected if the circadian clock has an ongoing active role in regulating sleep-wake (Deurveilher et al., 2002; Schwartz et al., 2011). Recent work has further confirmed that the circadian regulation of wakefulness is modulated by the SCN-PVN-lateral hypothalamic (LH) pathway (Ono et al., 2020). Activation of LH-GABA neurons can exert direct synaptic control over the sleep-promoting-galaninergic neurons in the ventrolateral preoptic nucleus (VLPO) promoting arousal during NREMS in the light phase (Venner et al., 2019). In addition, a LH-thalamic reticular nucleus (TRN)-GABAergicthalamocortical inhibitory circuit may be involved in the rapid arousal during NREMS-wake transitions (Herrera et al., 2016). Similarly, the loss of the widely projecting orexin/hypocretin neurons results in more frequent transitions into sleep and so would prevent prolongation of wake episodes (Hara et al., 2001). It may be that changes in GABAergic/glutamatergic drive impose the rapid changes in state, whereas neuropeptides such as galanin/orexin act as neuromodulators influencing the stabilization of sleep-wake states, and low (no?) amplitude and/or phasing of output from these cells underlie the sleep phenotypes in Cry1, Cry2-null, which are ameliorated following rescue of rhythmicity in the $\mathrm{SCN}^{\mathrm{Cry1}}$ mice (Willie et al., 2003; Herrera et al., 2016; Venner et al., 2019). In addition, the SCN may also act indirectly; initiation of behavioral rhythmicity in $\mathrm{SCN}^{\mathrm{Cry} 1}$ mice will in turn regulate their metabolic demands, providing feedback from the periphery and/or brain regions. These could in turn influence, indirectly, the timing and homeostatic regulation of sleep (Ehlen et al., 2017; Northeast et al., 2020). For example, overexpression of BMAL1 in skeletal muscle (but not the brain) is reported to influence the daily amount of NREMS, but not the 24 -h pattern to sleep/wake, nor the homeostatic responses to SD (Ehlen et al., 2017).

Sleep-dependent memory was severely compromised in $\mathrm{SCN}^{\mathrm{Con}}$ mice, consistent with other reports of cognitive impairment in Cry1/Cry2-nulls (Van der Zee et al., 2008; De Bundel et al., 2013). This could be a result of arrhythmia (in the SCN and/ or hippocampal formation), or a non-circadian, molecular consequence of local Cry deficiency. Restoration in $\mathrm{SCN}^{\mathrm{Cry} 1}$ mice refuted the latter, emphasizing the central importance of circadian organization to cognitive function, be it in the SCN and/or locally in the hippocampal formation, and driven by the SCN. The role of the SCN may, however, be bivalent. In hamsters made arrhythmic using a light pulse paradigm, memory was impaired (Ruby et al., 2008), but the effect was reversed by SCN ablation (Fernandez et al., 2014), suggesting that a dysfunctional SCN signal is more cognitively debilitating than no signal at all. Similarly, in a mouse model of down syndrome, impaired object recognition is restored by SCN ablation (Chuluun et al., 2020). These observations raise the possibility that cognitive deficits might be mitigated by improving circadian amplitude when it is disrupted as, for example, in patients with Alzheimer's disease (Hatfield et al., 2004; Leng et al., 2019).

In conclusion, by adopting a gain-of-function approach, we have shown that the suprachiasmatic clockwork can impose temporal organization on the sleep-wake cycle, facilitating circadian initiation and maintenance of wake, promoting sleep 
consolidation, the dynamics of homeostatic recovery, and sleepdependent memory. Thus, expression of Cry proteins outside the SCN region is not necessary to sustain these processes. Our results therefore add to understanding of the relative contributions of the SCN, extra-SCN clocks and circadian clock genes in the temporal organization of sleep and wake.

\section{References}

Berson DM, Dunn FA, Takao M (2002) Phototransduction by retinal ganglion cells that set the circadian clock. Science 295:1070-1073.

Borbély AA, Achermann P (1999) Sleep homeostasis and models of sleep regulation. J Biol Rhythms 14:557-568.

Borbély AA, Daan S, Wirz-Justice A, Deboer T (2016) The two-process model of sleep regulation: a reappraisal. J Sleep Res 25:131-143.

Brancaccio M, Edwards MD, Patton AP, Smyllie NJ, Chesham JE, Maywood ES, Hastings MH (2019) Cell-autonomous clock of astrocytes drives circadian behavior in mammals. Science 363:187-192.

Chuluun B, Pittaras E, Hong H, Fisher N, Colas D, Ruby NF, Heller HC (2020) Suprachiasmatic lesions restore object recognition in down syndrome model mice. Neurobiol Sleep Circadian Rhythms 8:100049.

Curie T, Mongrain V, Dorsaz S, Mang GM, Emmenegger Y, Franken P (2013) Homeostatic and circadian contribution to EEG and molecular state variables of sleep regulation. Sleep 36:311-323.

De Bundel D, Gangarossa G, Biever A, Bonnefont X, Valjent E (2013) Cognitive dysfunction, elevated anxiety, and reduced cocaine response in circadian clock-deficient cryptochrome knockout mice. Front Behav Neurosci 7:152.

Deboer T, Vansteensel MJ, Détári L, Meijer JH (2003) Sleep states alter activity of suprachiasmatic nucleus neurons. Nat Neurosci 6:1086-1090.

Deboer T, Détári L, Meijer JH (2007) Long term effects of sleep deprivation on the mammalian circadian pacemaker. Sleep 30:257-262.

Deurveilher S, Burns J, Semba K (2002) Indirect projections from the suprachiasmatic nucleus to the ventrolateral preoptic nucleus: a dual tract-tracing study in rat. Eur J Neurosci 16:1195-1213.

Dijk DJ, Archer SN (2010) PERIOD3, circadian phenotypes, and sleep homeostasis. Sleep Med Rev 14:151-160.

Easton A, Meerlo P, Bergmann B, Turek FW (2004) The suprachiasmatic nucleus regulates sleep timing and amount in mice. Sleep 27:1307-1318.

Edwards MD, Brancaccio M, Chesham JE, Maywood ES, Hastings MH (2016) Rhythmic expression of cryptochrome induces the circadian clock of arrhythmic suprachiasmatic nuclei through arginine vasopressin signaling. Proc Natl Acad Sci USA 113:2732-2737.

Ehlen JC, Brager AJ, Baggs J, Pinckney L, Gray CL, DeBruyne JP, Esser KA, Takahashi JS, Paul KN (2017) Bmall function in skeletal muscle regulates sleep. Elife 6:e26557.

Fernandez F, Lu D, Ha P, Costacurta P, Chavez R, Heller HC, Ruby NF (2014) Circadian rhythm. Dysrhythmia in the suprachiasmatic nucleus inhibits memory processing. Science 346:854-857.

Franken P, Thomason R, Heller HC, O'Hara BF (2007) A non-circadian role for clock-genes in sleep homeostasis: a strain comparison. BMC Neurosci $8: 87$.

Fuller PM, Lu J, Saper CB (2008) Differential rescue of light- and foodentrainable circadian rhythms. Science 320:1074-1077.

Gillette MU (2004) Does the SCN regulate more than sleep timing? Sleep 27:1240-1241.

Hamnett R, Chesham JE, Maywood ES, Hastings MH (2021) The cell-autonomous clock of VIP receptor VPAC2 cells regulates period and coherence of circadian behavior. J Neurosci 41:502-512.

Hara J, Beuckmann CT, Nambu T, Willie JT, Chemelli RM, Sinton CM, Sugiyama F, Yagami K, Goto K, Yanagisawa M, Sakurai T (2001) Genetic ablation of orexin neurons in mice results in narcolepsy, hypophagia, and obesity. Neuron 30:345-354.

Hasan S, van der Veen DR, Winsky-Sommerer R, Dijk DJ, Archer SN (2011) Altered sleep and behavioral activity phenotypes in PER3-deficient mice. Am J Physiol Regul Integr Comp Physiol 301:R1821-R1830.

Hastings MH, Maywood ES, Brancaccio M (2018) Generation of circadian rhythms in the suprachiasmatic nucleus. Nat Rev Neurosci 19:453-469.

Hatfield CF, Herbert J, van Someren EJ, Hodges JR, Hastings MH (2004) Disrupted daily activity/rest cycles in relation to daily cortisol rhythms of home-dwelling patients with early Alzheimer's dementia. Brain 127:1061-1074
Hattar S, Liao HW, Takao M, Berson DM, Yau KW (2002) Melanopsin-containing retinal ganglion cells: architecture, projections, and intrinsic photosensitivity. Science 295:1065-1070.

Hattar S, Lucas RJ, Mrosovsky N, Thompson S, Douglas RH, Hankins MW, Lem J, Biel M, Hofmann F, Foster RG, Yau KW (2003) Melanopsin and rod-cone photoreceptive systems account for all major accessory visual functions in mice. Nature 424:76-81.

Herrera CG, Cadavieco MC, Jego S, Ponomarenko A, Korotkova T, Adamantidis A (2016) Hypothalamic feedforward inhibition of thalamocortical network controls arousal and consciousness. Nat Neurosci 19:290-298.

Lang N, Rothkegel H, Reiber H, Hasan A, Sueske E, Tergau F, Ehrenreich H, Wuttke W, Paulus W (2011) Circadian modulation of GABA-mediated cortical inhibition. Cereb Cortex 21:2299-2306.

Laposky A, Easton A, Dugovic C, Walisser J, Bradfield C, Turek F (2005) Deletion of the mammalian circadian clock gene BMAL1/Mop3 alters baseline sleep architecture and the response to sleep deprivation. Sleep 28:395-409.

Lazar AS, Lazar ZI, Dijk DJ (2015) Circadian regulation of slow waves in human sleep: topographical aspects. Neuroimage 116:123-134.

Leng Y, Musiek ES, Hu K, Cappuccio FP, Yaffe K (2019) Association between circadian rhythms and neurodegenerative diseases. Lancet Neurol 18:307-318.

Maywood ES, Drynan L, Chesham JE, Edwards MD, Dardente H, Fustin JM, Hazlerigg DG, O'Neill JS, Codner GF, Smyllie NJ, Brancaccio M, Hastings MH (2013) Analysis of core circadian feedback loop in suprachiasmatic nucleus of mCryl-luc transgenic reporter mouse. Proc Natl Acad Sci USA 110:9547-9552.

Maywood ES, Elliott TS, Patton AP, Krogager TP, Chesham JE, Ernst RJ, Beránek V, Brancaccio M, Chin JW, Hastings MH (2018) Translational switching of Cryl protein expression confers reversible control of circadian behavior in arrhythmic Cry-deficient mice. Proc Natl Acad Sci USA 115:E12388-E12397.

Maywood ES, Chesham JE, Winsky-Sommerer R, Smyllie NJ, Hastings MH (2021) Circadian chimeric mice reveal an interplay between the suprachiasmatic nucleus and local brain clocks in the control of sleep and memory. Front Neurosci 15:639281.

Mistlberger RE (2005) Circadian regulation of sleep in mammals: role of the suprachiasmatic nucleus. Brain Res Brain Res Rev 49:429-454.

Naylor E, Bergmann BM, Krauski K, Zee PC, Takahashi JS, Vitaterna MH, Turek FW (2000) The circadian clock mutation alters sleep homeostasis in the mouse. J Neurosci 20:8138-8143.

Northeast RC, Vyazovskiy VV, Bechtold DA (2020) Eat, sleep, repeat: the role of the circadian system in balancing sleep-wake control with metabolic need. Curr Opin Physiol 15:183-191.

Ono D, Mukai Y, Hung CJ, Chowdhury S, Sugiyama T, Yamanaka A (2020) The mammalian circadian pacemaker regulates wakefulness via CRF neurons in the paraventricular nucleus of the hypothalamus. Sci Adv 6: eabd0384.

Palchykova S, Winsky-Sommerer R, Meerlo P, Dürr R, Tobler I (2006) Sleep deprivation impairs object recognition in mice. Neurobiol Learn Mem 85:263-271.

Patton AP, Edwards MD, Smyllie NJ, Hamnett R, Chesham JE, Brancaccio M, Maywood ES, Hastings MH (2020) The VIP-VPAC2 neuropeptidergic axis is a cellular pacemaking hub of the suprachiasmatic nucleus circadian circuit. Nat Commun 11:3394.

Paxinos G, Franklin KBJ (2001) The Mouse Brain in Stereotaxic Coordinates. San Diego, CA: Academic Press.

Ruby NF, Hwang CE, Wessells C, Fernandez F, Zhang P, Sapolsky R, Heller HC (2008) Hippocampal-dependent learning requires a functional circadian system. Proc Natl Acad Sci USA 105:15593-15598.

Saper CB, Scammell TE, Lu J (2005) Hypothalamic regulation of sleep and circadian rhythms. Nature 437:1257-1263.

Saper CB, Fuller PM, Pedersen NP, Lu J, Scammell TE (2010) Sleep state switching. Neuron 68:1023-1042.

Schmidt C, Collette F, Leclercq Y, Sterpenich V, Vandewalle G, Berthomier P, Berthomier C, Phillips C, Tinguely G, Darsaud A, Gais S, Schabus M, Desseilles M, Dang-Vu TT, Salmon E, Balteau E, Degueldre C, Luxen A, Maquet P, Cajochen C, et al. (2009) Homeostatic sleep pressure and responses to sustained attention in the suprachiasmatic area. Science 324:516-519. 
Schwartz MD, Urbanski HF, Nunez AA, Smale L (2011) Projections of the suprachiasmatic nucleus and ventral subparaventricular zone in the Nile grass rat (Arvicanthis niloticus). Brain Res 1367:146-161.

Shiromani PJ, Xu M, Winston EM, Shiromani SN, Gerashchenko D, Weaver DR (2004) Sleep rhythmicity and homeostasis in mice with targeted disruption of mPeriod genes. Am J Physiol Regul Integr Comp Physiol 287:R47-R57.

Sujino M, Masumoto KH, Yamaguchi S, van der Horst GT, Okamura H, Inouye ST (2003) Suprachiasmatic nucleus grafts restore circadian behavioral rhythms of genetically arrhythmic mice. Curr Biol 13:664-668.

Takahashi JS (2017) Transcriptional architecture of the mammalian circadian clock. Nat Rev Genet 18:164-179.

Tobler I, Borbély AA, Groos G (1983) The effect of sleep deprivation on sleep in rats with suprachiasmatic lesions. Neurosci Lett 42:49-54.

Tsai JW, Hannibal J, Hagiwara G, Colas D, Ruppert E, Ruby NF, Heller HC, Franken P, Bourgin P (2009) Melanopsin as a sleep modulator: circadian gating of the direct effects of light on sleep and altered sleep homeostasis in Opn4(-/-) mice. PLoS Biol 7:e1000125.

van der Horst GT, Muijtjens M, Kobayashi K, Takano R, Kanno S, Takao M, de Wit J, Verkerk A, Eker AP, van Leenen D, Buijs R, Bootsma D, Hoeijmakers JH, Yasui A (1999) Mammalian Cry1 and Cry2 are essential for maintenance of circadian rhythms. Nature 398:627-630.
Van der Zee EA, Havekes R, Barf RP, Hut RA, Nijholt IM, Jacobs EH, Gerkema MP (2008) Circadian time-place learning in mice depends on Cry genes. Curr Biol 18:844-848.

Venner A, De Luca R, Sohn LT, Bandaru SS, Verstegen AMJ, Arrigoni E, Fuller PM (2019) An inhibitory lateral hypothalamic-preoptic circuit mediates rapid arousals from sleep. Curr Biol 29:4155-4168.e5.

Willie JT, Chemelli RM, Sinton CM, Tokita S, Williams SC, Kisanuki YY, Marcus JN, Lee C, Elmquist JK, Kohlmeier KA, Leonard CS, Richardson JA, Hammer RE, Yanagisawa M (2003) Distinct narcolepsy syndromes in orexin receptor-2 and orexin null mice: molecular genetic dissection of non-REM and REM sleep regulatory processes. Neuron 38:715-730.

Wisor JP, O’Hara BF, Terao A, Selby CP, Kilduff TS, Sancar A, Edgar DM, Franken P (2002) A role for cryptochromes in sleep regulation. BMC Neurosci 3:20.

Wisor JP, Pasumarthi RK, Gerashchenko D, Thompson CL, Pathak S, Sancar A, Franken P, Lein ES, Kilduff TS (2008) Sleep deprivation effects on circadian clock gene expression in the cerebral cortex parallel electroencephalographic differences among mouse strains. J Neurosci 28:7193-7201.

Yu X, Zecharia A, Zhang Z, Yang Q, Yustos R, Jager P, Vyssotski AL, Maywood ES, Chesham JE, Ma Y, Brickley SG, Hastings MH, Franks NP, Wisden W (2014) Circadian factor BMAL1 in histaminergic neurons regulates sleep architecture. Curr Biol 24:2838-2844. 